\title{
The Impact of Buoyancy on Front Spreading in - Heterogeneous Porous Media in Two-Phase s Immiscible Flow
}

Diogo Bolster, ${ }^{1}$, Insa Neuweiler, ${ }^{2}$, Marco Dentz,,${ }^{3}$, Jesus Carrera ${ }^{3}$

${ }^{1}$ Environmental Fluid Dynamics

Laboratories, Department of Civil

Engineering and Geological Sciences,

University of Notre Dame, Notre Dame,

USA

${ }^{2}$ Institute for Fluid Mechanics and

Environmental Physics in Civil Engineering,

Hannover, Germany

${ }^{3}$ Institute of Environmental Analysis and

Water Studies (IDAEA), CSIC, Barcelona,

Spain 
$\mathrm{X}-2$

BOLSTER ET AL.: BUOYANT HETEROGENEOUS MULTIPHASE

${ }_{4}$ Abstract.

${ }_{5} \quad$ We study the influence of buoyancy and spatial heterogeneity on the spread-

${ }_{6}$ ing of the saturation front of a displacing fluid during injection into a porous

7 medium saturated with another, immiscible fluid. To do so we use a stochas-

s tic modeling framework. We derive an effective large-scale flow equation for

9 the saturation of the displacing fluid that is characterized by six nonlocal

flux terms, four that resemble dispersive type terms and two that have the

appearance of advection terms. From the effective large scale flow equation

we derive measures for the spreading of the saturation front. A series of full

two phase numerical solutions are conducted to compliment the analytical

developments. We find that the interplay between density and heterogene-

ity leads to an enhancement of the front spreading on one hand and to a renor-

malization of the evolution of the mean front position compared with an equivan important role in several applications, including for example carbon sequestration and enhanced oil recovery . 


\section{Introduction}

Capturing the influence of physical heterogeneity on flow and transport in geological media is still one of the great challenges facing us today. Even for linear problems, such as single phase flow and transport many questions remain unanswered and while many have been presented with some success, no single clear model has emerged as capable of capturing all effects of heterogeneity (see [e.g., Dagan, 1989; Gelhar, 1993; Neuman and Tartakovsky, 2009]. Similarly, accounting for the influence of buoyancy on single phase flow [e.g., Henry, 1964; Kalejaiye and Cardoso, 2005; Huppert and Woods, 1995; Dentz et al., 2006] and transport [e.g., Graf and Therrien, 2008; Bolster et al., 2007] in porous media is a challenging problem that has a rich body of work dedicated to it.

Many interesting and relevant problems in porous media involve the flow and interaction of two immiscible fluids. Relevant examples that receive much attention include CO2 sequestration [e.g., Bachu, 2008; Bachu and Adams, 2003; Bryant et al., 2008; Riaz and Tchelepi, 2008] and enhanced oil recovery [e.g., Lake, 1989; Ferguson et al., 2009; Dong et al., 2009; Tokunaga et al., 2000]. Accounting for the effects of mobility (viscosity differences between phases) and capillarity introduces significant complexity and results in highly nonlinear and coupled governing equations [e.g., Binning and Celia, 1999]. Add to this buoyancy effects when the two phases are of differing density and one has a very interesting and challenging problem (even in the absence of heterogeneity).

In this work we focus on the interaction of buoyancy and heterogeneity effects on multiphase flows. To do so, we consider a displacement problem where an invading phase displaces another one as depicted in Figure 1. We neglect the influence of capillarity 
${ }_{41}$ by using the commonly used Buckley-Leverett approximation, which we discuss in more

${ }_{42}$ detail in Section 2. In such a displacement problem there is typically a sharp interface

between the invading and displaced phases. Spatial variability in the flow field, induced

by heterogeneity, cause this sharp interface to vary in space, which results in spreading of the front. At the same time buoyancy plays its role. In the case of a stable displacement, the spreading ultimately induces lateral pressure gradients that slow down the spreading of the interface. Similarly, an unstable injection will result in greater spreading due to buoyancy. This is illustrated clearly in Figure 1 where the results of three numerical simulations are presented, one with no buoyancy effects (Fig. 1a), one with stabilizing buoyancy (Fig. 1b) and one with destabilizing buoyancy (Fig. 1c).

To date, in the field of single phase flows, the approaches to capture the effect of heterogeneity that have achieved most success are stochastic methods. The theory of such approaches is described extensively in the literature [e.g., Dagan, 1989; Brenner and Edwards, 1993; Gelhar, 1993; Rubin, 2003]. In the context here, if one averages transversely across the transition zones depicted in Figure 1, the resulting transition zone between high and low saturation of the displacing fluid can have the appearance of a dispersive mixing zone. It should of course be noted that this averaged dispersive zone does not represent actual mixing as only spreading occurs. However, for appilcations where the fluid-fluid interfacial area is important, it is important to have model predictions that quantify the spreading zone.

Dispersive transition zones in solute transport problems have typically been characterized by spatial moments and a wide body of literature exists doing so [e.g., Aris, 1956; Gelhar and Axness, 1983; Dagan, 1989; Kitanidis, 1988; Dentz and Carrera, 2007; Bolster 
64

et al., 2009b]. Similar approaches have been applied to two-phase flow, but most work along these lines has been limited to horizontal displacements that neglect buoyancy effects. Cvetovic and Dagan [1996] and Dagan and Cvetkovic [1996] applied a Lagrangian perturbation theory approach in order to determine the averaged cumulative recovery of the displacing fluid and the spatial moments of the fluid distribution. They found that the heterogeneities cause a dispersive growth of the second moment. However, they did not quantify it. Similarly Zhang and Tchelepi [1999] found a dispersion effect for the immiscible displacement in the horizontal direction. This dispersion coefficient was calculated semi-analytically by numerical means by Langlo and Espedal [1995], who also applied a perturbation theory approach. Their approach was extended by Neuweiler et al. [2003] to quantify the dispersion coefficient analytically and later by Bolster et al. [2009a] to include temporal fluctuations in the flow field. Within the validity of perturbation theory and in direct analogy to single phase flow, they showed that the dispersive growth for neutrally stable displacement was directly proportional to the variance and the correlation length of the permeability field. As such a natural question arises: given the additional influence of buoyancy, can we anticipate the same behavior?

For vertical immiscible displacement in the presence of buoyancy effects we anticipate a similar quasi-dispersive transition zone of the averaged front, which will be augmented or suppressed due to buoyancy. The heterogeneity still leads to fluctuations in the velocity field as illustrated in Figure 1. However, the process will be more complicated and not solely due to the stabilizing and destabilizing processes mentioned above. After all, such stabilization/destabilization effects will occur even for single phase miscible displacement 
${ }_{86}$ [e.g., Welty and Gelhar, 1991; Kempers and Haas, 1994]), leading to the question what

${ }_{87}$ additional role the multiphase nature of this flow plays?

88

In the absence of buoyancy effects the Buckley Leveret problem is governed by a single dimensionless parameter, which is the viscosity ratio (or ratio of the viscosities of the two phases). This dimensionless number does not depend on any of the parameters associated with the flow or porous medium. This means that while heterogeneity in the porous medium induces fluctuations in the flow field it does not affect the fundamental fluid properties in an equivalent homogeneous medium. Thus, the (mean) front positions obtained from the solutions of the homogeneous and heterogeneous media are identical.

On the other hand, when one includes buoyancy effects, a second dimensionless number is necessary to describe the system, namely the gravity number. The gravity number physically reflects the ratio of buoyancy to viscous forces. The buoyancy number (defined formally and discussed further in Section 2) is directly proportional to the permeability of the porous medium. Therefore when the permeability field is heterogeneous in space, so too is the buoyancy number. This means that while the viscosity ratio is insensitive to heterogeneity, the gravity number can potentially vary over orders of magnitude depending on how variable the permeability field is. This raises another important and potentially problematic question: as this system is so inherently nonlinear, does the arithmetic mean (or for that matter any other mean) of the gravity number provide a good representative measure of the behavior of the heterogeneous system?

In fact, as the buoyancy number varies in space, in a manner directly proportional to the spatial variations in permeability one might anticipate a local contribution to the dispersion front spreading effect beyond the nonlocal contribution that arises from 
fluctuations in the velocity field. In this paper we aim to answer the following questions regarding buoyancy influenced multiphase immiscible displacement in a heterogeneous medium.

1. Can we, using perturbation theory, asses the rate of front spreading that occurs?

2. What measures of the heterogeneous field (e.g. variance, correlation length) control this spreading? Also, why and how do they?

3. What influence does the heterogeneity in gravity number have? And does the arithmetic mean of the gravity number represent a mean behavior in the heterogeneous system considering that the problems considered here are highly non-linear?

\section{Model}

The flow of two immiscible fluids in a porous medium can be described by conservation of mass and momentum. Momentum conservation is expressed by the Darcy law, which is

$$
\mathbf{q}^{(j)}(\mathbf{x}, t)=-\frac{k(\mathbf{x}) k_{r j}\left(S_{j}\right)}{\mu_{j}}\left[\nabla p_{j}(\mathbf{x}, t)+\rho_{j} g \mathbf{e}_{1}\right],
$$

where $\mathbf{q}^{(j)}(\mathbf{x}, t)$ and $p_{j}(\mathbf{x}, t)$ are specific discharge and pressure of fluid $j, \mu_{j}$ and $\rho_{j}$ are viscosity and density of fluid $j, k(\mathbf{x})$ is the intrinsic permeability of the porous medium, $k_{r j}\left[S_{j}(\mathbf{x}, t)\right]$ is the relative permeability of phase $j$ (which depends on saturation). The 1-direction of the coordinate system is aligned with negative gravity acceleration as expressed by $\mathbf{e}_{1}$, which denotes the unit-vector in the 1-direction. Mass conservation for each fluid is given by [e.g., Bear, 1988]

$$
\frac{\partial}{\partial t} \omega \rho_{j} S_{j}(\mathbf{x}, t)+\nabla \cdot \rho_{j} \mathbf{q}^{(j)}(\mathbf{x}, t)=0
$$


We assume here that the medium and the fluid are incompressible so that porosity $\omega$ and density $\rho_{j}$ of each fluid are constant. The saturations $S_{j}$ of each fluid sum up to one and the difference of the pressures in each fluid defines the capillary pressure $p_{c}(S)$

$$
S_{n w}+S_{w}=1, \quad p_{n w}-p_{w}=p_{c}\left(S_{n w}\right),
$$

where $j=n w$ indicates the non-wetting fluid and $j=w$ the wetting fluid. In the problem studied here we will use two phases $j=i, d$, where $i$ refers to an injected phase and $d$ to a displaced phase. From here on, $S$ refers to the saturation of the injected phase $S_{i}$. From the incompressibility conditions and mass conservation, it follows that the divergence of the total specific discharge $\mathbf{Q}(\mathbf{x}, t)=\mathbf{q}^{(i)}(\mathbf{x}, t)+\mathbf{q}^{(d)}(\mathbf{x}, t)$ is zero

$$
\nabla \cdot \mathbf{Q}(\mathbf{x}, t)=0 .
$$

Eliminating $\mathbf{q}^{(i)}(\mathbf{x}, t)$ from Eq. (2) in favor of $\mathbf{Q}(\mathbf{x}, t)$, one obtains [Bear, 1988]

$$
\frac{\partial S}{\partial t}+\nabla \cdot\left[\mathbf{Q} f(S)+\frac{k \Delta \rho g}{\mu_{d}} \mathbf{e}_{1} g(S)\right]-\nabla \cdot\left[f(S) k \frac{k_{r d}(S)}{\mu_{d}} \frac{\mathrm{d} p_{c}(S)}{\mathrm{d} S} \nabla S\right]=0,
$$

where $\Delta \rho=\rho_{d}-\rho_{i}$. We set $\omega=1$ for simplicity (which is equivalent to rescaling time). The fractional flow function $f(S)$ and modified fractional flow function $g(S)$ are defined by

$$
f(S)=\frac{k_{r i}(S)}{k_{r i}(S)+M k_{r d}(S)}, \quad g(S)=k_{r d} f(S) .
$$

where the viscosity ratio $M$ is defined by

$$
M=\frac{\mu_{i}}{\mu_{d}}
$$

In this work we consider the commonly studied problem of one fluid displacing another immiscible one. We focus on fluid movement in a vertical two-dimensional porous medium which is initially filled with fluid $d$. As outlined above, the 1-axis points upwards. Fluid 
143

$i$ is injected along a horizontal line at a constant volumetric flux $\bar{Q}$, displacing fluid $d$. We consider flow far away from the domain boundaries and thus disregard boundary effects. The resulting mean pressure gradient is then aligned with the 1-direction of the coordinate system. We restrict our focus on flows where capillary pressure effects are small and thus we neglect them. The approximation to neglect capillary forces implies thus displacement processes on large length scales, such as that of an oil reservoir, are considered and that the flow rates are high. The approximation neglects the influence of small scale heterogeneity of the capillary entry pressure (e.g. Neuweiler et al. [2010]). This might be questionable if residual saturations and macroscopic trapping would be important. However, as the focus of this paper is the spreading of immiscible displacement fronts in geotechnical applications, we proceed by neglecting these effects. This problem of immiscible two phase viscous dominated flow is commonly known as the Buckley-Leverett problem. Unlike many previous studies we include the influence of buoyancy.

We define the dimensionless coordinates, time and total flow by

$$
x_{i}=l \tilde{x}_{i}, \quad \quad t=\tau_{Q} \tilde{t}, \quad \mathbf{Q}=\tilde{\mathbf{Q}} \bar{Q},
$$

where $l$ is a characteristic length scale such as the length of the domain and the advection scale $\tau_{Q}$ is defined by $\tau_{Q}=l / \bar{Q}$. In the following $l$ will be set equal to the correlation scale of the permeability field $k(\mathbf{x})$. The governing equation reads in non-dimensional terms as

$$
\frac{\partial S}{\partial \tilde{t}}+\tilde{\nabla} \cdot \tilde{\mathbf{Q}} f(S)+\frac{\partial}{\partial x_{1}} N g(S),=0
$$

where we disregard the capillary diffusion term, conform with the Buckley-Leverett approximation. We define the (dimensionless) gravity number $N$ by 


$$
N=\frac{k \Delta \rho g}{\mu_{d} \bar{Q}} .
$$

It compares buoyancy forces to forces driving the movement of the front. A positive gravity number implies a less dense fluid displacing a denser one, a negative gravity number vice versa. Note that the gravity number is spatially variable because the permeability $k$ is spatially variable. For convenience, in the following the tildes will be dropped and all quantities are understood to be dimensionless.

\section{Homogeneous Solution}

In order to study the heterogeneous problem it is important to explore and understand the homogeneous one, that is, for constant permeability, $k=$ constant. In this case, Eq. (9) simplifies to

$$
\frac{\partial S_{h}}{\partial t}+\frac{\partial f}{\partial x_{1}}+N \frac{\partial g}{\partial x_{1}}=0
$$

where $S_{h}$ is the homogeneous saturation. The solution of this problem is governed by two dimensionless quantities, namely the viscosity ratio $M$ and the gravity number $N$. Both these numbers determine the form of the solution of (11). Eq. (11) can be solved using the method of characteristics [e.g., Marle, 1981]. The velocity of the characteristics of constant saturation are given by the derivatives of the total fractional flow function $\phi(S)$

$$
\phi\left(S_{h}\right)=f\left(S_{h}\right)+N g\left(S_{h}\right)
$$

Owing to the hyperbolic nature of equation (11) the solution has a sharp front that travels with the front velocity $Q^{f}$. It can be written in the scaling form

$$
S_{h}\left(x_{1} / t\right)=S_{h}^{r}\left(x_{1} / t\right) H\left(1-\frac{x_{1}}{Q^{f t}}\right)
$$


where $H(x)$ is the Heaviside step function. The front position is given by $x_{f}(t)=Q^{f} t$.

The front velocity is

$$
Q^{f}=\frac{\mathrm{d} \phi\left(S_{h}^{f}\right)}{d S_{h}}
$$

where the front saturation $S_{h}^{f}$ can be determined by the Welge tangent method [e.g., Marle, 1981], which states that

$$
\frac{\mathrm{d} \phi\left(S_{h}^{f}\right)}{\mathrm{d} S_{h}^{f}}=\frac{\phi\left(S_{h}^{f}\right)}{S_{h}^{f}} .
$$

This implies together with (14) that the front velocity is given by $Q^{f}=\phi\left(S_{h}^{f}\right) / S_{h}^{f}$.

The form of the rear saturation $S^{r}$ is obtained by the method of characteristics. As outlined above, the characteristic velocities behind the front are given by $\mathrm{d} \phi\left(S_{h}^{r}\right) / \mathrm{d} S_{h}^{r}$. As iso-saturation points travel with constant velocity, the characteristic velocity at a given point $x_{1}$ and time $t$ is

$$
\frac{x_{1}}{t}=\frac{\mathrm{d} \phi\left(S_{h}^{r}\right)}{\mathrm{d} S^{r}}
$$

The rear saturation is obtained by inverting this relation.

\subsection{Homogeneous saturation profiles}

For negative gravity numbers, when the density of the injected phase is greater than that of the displaced phase, the total fractional flow function $\phi$ may not be a monotonically increasing function and may have a maximum between the front and maximum saturations. This causes the derivative $\mathrm{d} \phi\left(S^{h}\right) / \mathrm{d} S^{h}$ to be negative for saturations larger than the saturation at which $\phi\left(S^{h}\right)$ is maximum. As $\mathrm{d} \phi\left(S^{h}\right) / \mathrm{d} S^{h}$ is the velocity at which zones of saturation $S^{h}$ move, this implies that saturation values larger than the value at which velocities turn negative would move in the direction opposite to the flow direction. In order to deal with these unphysical characteristics, a procedure similar to the one to 
can similarly induce gravitational instabilities [e.g. Noetinger et al., 2004]. A criterion for these instabilities in outlined in section 3.2

The location of the front may be analyzed by looking at the derivative of the saturation field as this has a sharp delta function at the front, which allows the quantification of spreading around it [Bolster et al., 2009a]. The expression for the derivative of saturation is given by

$$
-\frac{\partial S_{h}}{\partial x_{1}}=-\frac{\partial S_{h}^{r}\left(x_{1} / t\right)}{\partial x_{1}} H\left(\frac{x_{1}}{Q^{f} t}-1\right)+\frac{1}{Q^{f} t} S^{r}\left(x_{1} / t\right) \delta\left(1-\frac{x_{1}}{Q^{f} t}\right)
$$

The derivatives of saturation for the profiles in Figure $2 \mathrm{~b}$ are shown in Figure 3. Here the delta function at the front is clearly illustrated.

\subsection{Stability of the solution}

The solution of (11) can become unstable. Both viscous and gravity forces have an impact on the stability of the solution. If the total viscosity $\left(k_{\mathrm{rel}, 1} / \mu_{1}+k_{\mathrm{rel}, 2} / \mu_{2}\right)$ directly behind of the front is greater than the total viscosity directly ahead of the front the interface becomes unstable [e.g., Saffman and Taylor, 1958; Riaz and Tchelepi, 2006].

On the other hand, for $\Delta \rho<0$ gravity tends to damp out perturbations to the interface if the displacing fluid is heavier than the displaced fluid. Conversely if $\Delta \rho>0$ any perturbation will be enhanced. A criterion for stability can be found by introducing a critical velocity [Noetinger et al., 2004]

$$
q_{\mathrm{crit}}=\frac{k S^{f} \Delta \rho g}{\mu_{d}\left(M_{\text {shock }}\left(S^{f}\right)^{-1}-1\right)},
$$

where

$$
M_{\text {shock }}(S)=\frac{\left.\left(k_{r i} / \mu_{i}+k_{r d} / \mu_{d}\right)\right|_{S=S_{f}^{f}}}{\left.\left(k_{r i} / \mu_{i}+k_{r d} / \mu_{d}\right)\right|_{S=0}} .
$$


234 trigger fingering or be counteracted if the flow is stabilizing.

\section{Large Scale Flow Model} for the front spreading due to fluctuations in the permeability field.

\subsection{Stochastic Model} about it,

$$
k(\mathbf{x})=\bar{k}[1+\kappa(\mathbf{x})]
$$

Their correlation function of the permeability fluctuations is

$$
\overline{\kappa(\mathbf{x}) \kappa\left(\mathbf{x}^{\prime}\right)}=C^{k k}\left(\mathbf{x}-\mathbf{x}^{\prime}\right)
$$

and unstable otherwise. In a heterogeneous medium the heterogeneities cause perturbations of the interface between the fluids. Depending on the stability criteria of the flow these perturbations can either be enforced or damped out. Thus heterogeneities can either

In the following, we derive large scale flow equations by stochastic averaging of the original local scale flow equation. This results in a large scale effective flow equation for the average saturation. In section 5, using this effective flow equation, we define measures

We employ a stochastic modeling approach in order to quantify the impact of medium heterogeneity on the saturation front of the displacing fluid. The spatial variability of the intrinsic permeability $k(\mathbf{x})$ is modeled as a stationary correlated stochastic process in space. Its constant mean value is $\overline{k(\mathbf{x})}=\bar{k}$, where the overbar denotes the ensemble average. We decompose the permeability into its mean and (normalized) fluctuations 
250

The variance and correlation length are defined by

$$
\sigma_{k k}^{2}=C^{k k}(\mathbf{0}), \quad l_{k k}^{2}=\frac{\int \mathrm{d}^{2} x C^{k k}(\mathbf{x})}{\sigma_{k k}^{2}} .
$$

For simplicity, we assume the the permeability is statistically isotropic. The gravity number (10) is a linear function of permeability. Using the decomposition (21), it is given by

$$
N(\mathbf{x})=\bar{N}[1+\kappa(\mathbf{x})]
$$

where the mean gravity number is given by

$$
\bar{N}=\frac{\bar{k} \Delta \rho g}{\mu_{d} \bar{Q}} .
$$

We consider injection of the displacing fluid at an injection plane perpendicular to the one-direction of the coordinate system. The boundary flux in dimensionless notation is equal to $\bar{Q}=1$. The spatial randomness is mapped onto the phase discharges and thus on the total discharge via the Darcy equations (1), which renders the total discharge a spatial random field as well. Due to the boundary conditions the (dimensionless) mean flow velocity is $\overline{\mathbf{Q}(\mathbf{x}, t)}=\mathbf{e}_{1}$. Thus, we can decompose the total flux into its (constant) mean value and fluctuations about it

$$
\mathbf{Q}(\mathbf{x}, t)=\mathbf{e}_{1}+\mathbf{q}^{\prime}(\mathbf{x}) .
$$

Note that $\mathbf{q}^{\prime}(\mathbf{x}, t)$ in principle depends on saturation. However, since it is driven by a constant boundary flux, it is a reasonable approach to consider the total flow velocity as independent of saturation. In particular, it is worth noting that this is a good assumption away from the front position. This is no longer valid close to the front [e.g., Neuweiler et al., 2003]. Thus, strictly speaking, the velocity fluctuations cannot be considered sta- 
tionary and thus the velocity correlation function is given by

$$
\overline{q_{i}^{\prime}(\mathbf{x}) q_{j}^{\prime}\left(\mathbf{x}^{\prime}\right)}=C_{i j}^{q q}\left(\mathbf{x}, \mathbf{x}^{\prime}\right)
$$

The cross-correlation between the velocity and permeability fluctuations are accordingly

$$
\overline{q_{i}^{\prime}(\mathbf{x}) \kappa\left(\mathbf{x}^{\prime}\right)}=C_{i}^{k q}\left(\mathbf{x}, \mathbf{x}^{\prime}\right)
$$

\subsection{Average Flow Equation}

In analogy to solute transport in heterogeneous media [e.g., Gelhar and Axness, 1983; Koch and Brady, 1987; Neuman, 1993; Cushman et al., 1994], the spread of the ensemble averaged saturation front $\overline{S(\mathbf{x}, t)} \equiv \bar{S}(\mathbf{x}, t)$ due to spatial heterogeneity is modeled by a non-Markovian effective equation. Note that the averaging equation is in general nonMarkovian [e.g., Zwanzig, 1961; Kubo et al., 1991; Koch and Brady, 1987; Cushman et al., 1994; Neuman, 1993], which is expressed by spatio-temporal non-local flux terms. Under certain conditions, these fluxes can be localized.

We follow the methodology routinely applied when deriving average dynamics [e.g., Koch and Brady, 1987; Neuman, 1993; Cushman et al., 1994; Tartakovsky and Neuman, 1998], which consists of (i) separating the saturation into mean and fluctuating components, (ii) establishing a (non-closed) system of equations for the average saturation and the saturation fluctuations, (iii) closing the system by disregarding terms that are of higherorder in the variance of the fluctuations of the underlying random fields.

Following (24) and (26), we also decompose the saturation into its ensemble mean and fluctuations about it

$$
S(\mathbf{x}, t)=\bar{S}(\mathbf{x}, t)+S^{\prime}(\mathbf{x}, t)
$$



in Section 6 also justify this approximation. for the saturation $S(\mathbf{x}, t)$ is given by

$$
\begin{aligned}
& \frac{\partial \bar{S}(\mathbf{x}, t)}{\partial t}+\frac{\partial S^{\prime}(\mathbf{x}, t)}{\partial t}+\frac{\partial f(\bar{S})}{\partial x_{1}}+\frac{\partial}{\partial x_{1}} \frac{\mathrm{d} f(\bar{S})}{\mathrm{d} \bar{S}} S^{\prime}(\mathbf{x}, t)+\bar{N} \frac{\partial}{\partial x_{1}} g(\bar{S}) \\
& +\bar{N} \frac{\partial}{\partial x_{1}} \frac{\mathrm{d} g(\bar{S})}{\mathrm{d} \bar{S}} S^{\prime}(\mathbf{x}, t)+\mathbf{q}^{\prime}(\mathbf{x}) \cdot \nabla f(\bar{S})+\bar{N} \frac{\partial}{\partial x_{1}} \kappa(\mathbf{x}, t) g(\bar{S}) \\
& =-\mathbf{q}^{\prime}(\mathbf{x}) \cdot \nabla \frac{\mathrm{d} f(\bar{S})}{\mathrm{d} \bar{S}} S^{\prime}(\mathbf{x}, t)-\bar{N} \frac{\partial}{\partial x_{1}} \kappa(\mathbf{x}) \frac{\mathrm{d} g(\bar{S})}{\mathrm{d} \bar{S}} S^{\prime}(\mathbf{x}, t) .
\end{aligned}
$$

Averaging the latter over the ensemble gives

$$
\begin{aligned}
& \frac{\partial \bar{S}(\mathbf{x}, t)}{\partial t}+\frac{\partial f(\bar{S})}{\partial x_{1}}+\bar{N} \frac{\partial g(\bar{S})}{\partial x_{1}} \\
& =-\nabla \cdot \overline{\mathbf{q}^{\prime}(\mathbf{x}) S^{\prime}(\mathbf{x}, t)} \frac{\mathrm{d} f(\bar{S})}{\mathrm{d} \bar{S}}-\bar{N} \frac{\partial}{\partial x_{1}} \overline{\kappa(\mathbf{x}) S^{\prime}(\mathbf{x}, t)} \frac{\mathrm{d} g(\bar{S})}{\mathrm{d} \bar{S}}
\end{aligned}
$$

In order to be consistent with the second-order perturbation analysis that follows, the above expressions should technically be expanded to second order. However, including these additional terms significantly complicates the analysis and previous work [e.g., Efendiev and Durlofsky, 2002; Neuweiler et al., 2003; Bolster et al., 2009a]) illustrates that these additional terms do not contribute significantly to the system in the absense of buoyancy effects. We disregarded them in the following and justify this a postriori by the agreement with numerical simulations in section 6 . The results of this work discussed

Using decompositions (24), (26) and (29) as well as (30) in (9), the local scale equation

Subtracting (32) from (31), we obtain an equation for the saturation fluctuations. However, this system of equations is not closed with respect to the average saturation. In 
where $G\left(\mathbf{x}, t \mid \mathbf{x}^{\prime}, t^{\prime}\right)$ solves

$$
\begin{aligned}
& \frac{\partial \bar{S}(\mathbf{x}, t)}{\partial t}+\frac{\partial f(\bar{S})}{\partial x_{1}}+\bar{N} \frac{\partial g(\bar{S})}{\partial x_{1}} \\
& -\nabla \cdot \int \mathrm{d} \mathbf{x}^{\prime} \int_{0}^{t} \mathrm{~d} t^{\prime} \mathcal{A}\left(\mathbf{x}, t \mid \mathbf{x}^{\prime}, t^{\prime}\right) g\left[\bar{S}\left(\mathbf{x}^{\prime}, t^{\prime}\right)\right] \\
& -\nabla \cdot \int \mathrm{d} \mathbf{x}^{\prime} \int_{0}^{t} \mathrm{~d} t^{\prime} \mathcal{D}^{(g)}\left(\mathbf{x}, t \mid \mathbf{x}^{\prime}, t^{\prime}\right) \nabla^{\prime} g\left[\bar{S}\left(\mathbf{x}^{\prime}, t^{\prime}\right)\right] \\
& -\nabla \cdot \int \mathrm{d} \mathbf{x}^{\prime} \int_{0}^{t} \mathrm{~d} t^{\prime} \mathcal{D}^{(f)}\left(\mathbf{x}, t \mid \mathbf{x}^{\prime}, t^{\prime}\right) \nabla^{\prime} f\left[\bar{S}\left(\mathbf{x}^{\prime}, t^{\prime}\right)\right]=0
\end{aligned}
$$

where the advection kernel $\mathcal{A}\left(\mathbf{x}, t \mid \mathbf{x}^{\prime}, t^{\prime}\right)$ is defined by

$$
\frac{\partial G\left(\mathbf{x}, t \mid \mathbf{x}^{\prime}, t^{\prime}\right)}{\partial t}+\frac{\partial}{\partial x_{1}} \frac{\mathrm{d} f(\bar{S})}{\mathrm{d} \bar{S}} G\left(\mathbf{x}, t \mid \mathbf{x}^{\prime}, t^{\prime}\right)+\bar{N} \frac{\partial}{\partial x_{1}} \frac{\mathrm{d} g(\bar{S})}{\mathrm{d} \bar{S}} G\left(\mathbf{x}, t \mid \mathbf{x}^{\prime}, t^{\prime}\right)=0
$$

for the initial condition $G\left(\mathbf{x}, t \mid \mathbf{x}^{\prime}, t^{\prime}\right)=\delta\left(\mathbf{x}-\mathbf{x}^{\prime}\right)$, zero boundary conditions at $x_{1}=0$ and $x_{1}=\infty$ and zero normal derivative at the horizontal boundaries. Inserting (34) into (32), we obtain a non-linear upscaled equation for the ensemble averaged saturation

$$
\begin{aligned}
c_{i}\left(\mathbf{x}, t \mid \mathbf{x}^{\prime}, t^{\prime}\right) & =\bar{N} \frac{\mathrm{d} f[\bar{S}(\mathbf{x}, t)]}{\mathrm{d} \bar{S}} G\left(\mathbf{x}, t \mid \mathbf{x}^{\prime}, t^{\prime}\right) \frac{\partial C_{i}^{k q}\left(\mathbf{x}, \mathbf{x}^{\prime}\right)}{\partial x_{1}^{\prime}} \\
& +\delta_{i 1} \bar{N}^{2} \frac{\mathrm{d} g[\bar{S}(\mathbf{x}, t)]}{\mathrm{d} \bar{S}} G\left(\mathbf{x}, t \mid \mathbf{x}^{\prime}, t^{\prime}\right) \frac{\partial C^{k k}\left(\mathbf{x}-\mathbf{x}^{\prime}\right)}{\partial x_{1}^{\prime}} .
\end{aligned}
$$


The dispersion kernels have four contributions in total, two of which are due to autocorrelations of the velocity and permeability fluctuations and two due to cross-correlations between them,

$$
\begin{aligned}
\mathcal{D}_{i j}^{(g)}\left(\mathbf{x}, t \mid \mathbf{x}^{\prime}, t^{\prime}\right) & =\delta_{j 1} \bar{N} \frac{\mathrm{d} f[\bar{S}(\mathbf{x}, t)]}{\mathrm{d} \bar{S}} G\left(\mathbf{x}, t \mid \mathbf{x}^{\prime}, t^{\prime}\right) C_{i}^{n q}\left(\mathbf{x}, \mathbf{x}^{\prime}\right) \\
& +\delta_{i 1} \delta_{j 1} \bar{N}^{2} \frac{\mathrm{d} g[\bar{S}(\mathbf{x}, t)]}{\mathrm{d} \bar{S}} G\left(\mathbf{x}, t \mid \mathbf{x}^{\prime}, t^{\prime}\right) C^{n n}\left(\mathbf{x}-\mathbf{x}^{\prime}\right) \\
\mathcal{D}_{i j}^{(f)}\left(\mathbf{x}, t \mid \mathbf{x}^{\prime}, t^{\prime}\right) & =\frac{\mathrm{d} f[\bar{S}(\mathbf{x}, t)]}{\mathrm{d} \bar{S}} G\left(\mathbf{x}, t \mid \mathbf{x}^{\prime}, t^{\prime}\right) C_{i j}^{q q}\left(\mathbf{x}, \mathbf{x}^{\prime}\right) \\
& +\delta_{i 1} \bar{N} \frac{\mathrm{d} g[\bar{S}(\mathbf{x}, t)]}{\mathrm{d} \bar{S}} G\left(\mathbf{x}, t \mid \mathbf{x}^{\prime}, t^{\prime}\right) C_{j}^{n q}\left(\mathbf{x}, \mathbf{x}^{\prime}\right) .
\end{aligned}
$$

The first contribution in (37c) quantifies the impact on the large scale flow behavior due to velocity fluctuations, which has been quantified in Bolster et al. [2009a]. The remaining terms reflect the added influence of buoyancy, which manifest themselves due to cross-correlation between velocity and permeability fluctuations.

Note that equation (36), the large scale flow equation for the mean saturation, has the structure of a non-linear advection-dispersion equation characterized by spatio-temporal non-local advective and dispersive fluxes. As outlined above, such non-local fluxes typically occur when averaging. While in the absence of buoyancy, the spatial heterogeneity gives rise to a non-linear and non-local dispersive flux, in the presence of buoyancy, there are additional contributions to this dispersive flux as well as disorder-induced contributions to the advective flux as quantified by the kernel $\mathcal{A}\left(\mathbf{x}, t \mid \mathbf{x}^{\prime}, t^{\prime}\right)$.

Note that the non-linear character of the two-phase problem is preserved during the upscaling exercise. The non-linearity of the problem is quasi-decoupled in terms of the Green function; Eq. (35) for $G\left(\mathbf{x}, t \mid \mathbf{x}^{\prime}, t^{\prime}\right)$ is linear but depends on the average saturation. 


\section{Quantification of Average Front Spreading by Apparent Dispersion}

In direct analogy to solute transport we will quantify the additional spreading that occurs due to heterogeneity by an apparent dispersion coefficient. It should be stressed that the apparent dispersion coefficient does not only capture effects due to an effective dispersion term in the averaged flow equation (36). The renormalized advective flux term quantified by the kernel (37a) also contributes to the evolution of the apparent dispersion coefficient as defined below.

\subsection{Spatial moments}

As done by Bolster et al. [2009a] we will study the influence on the derivative of the saturation, given by

$$
\bar{s}(\mathbf{x}, t)=-L^{-1} \frac{\partial \bar{S}(\mathbf{x}, t)}{\partial x_{1}} .
$$

where $L$ is the horizontal extension of the flow domain. Recall that fluid is injected over the whole medium cross-section. The motivation for this is that the homogeneous solution develops a shock front, which is captured sharply by measuring the derivative. The resulting averaged profile under the influence of heterogeneity has an appearance similar to a Gaussian type bell that diffuses about this sharp delta function (much like a point injection in the case of single phase solute transport). The goal is to quantify the spreading of the averaged front of $\bar{S}(x, t)$ by the width of the averaged profile of $\bar{s}_{i}(x, t)$. For an illustration see Figure 9.

In analogy to the definition of the width of a tracer plume by spatial moments, we will analyze the spatial moments of $\bar{s}(x, t)$. Let us define the first and second moments in 
341

direction of the mean flow by

$$
m_{1}^{(1)}(t)=\int d^{2} x x_{1} s(\mathbf{x}, t), \quad m_{11}^{(2)}(t)=\int d^{d} x_{1}^{2} s(\mathbf{x}, t)
$$

The second centered moment

$$
\kappa_{11}(t)=m_{11}^{(1)}(t)-m_{1}^{(2)}(t)^{2} .
$$

describes the width of the saturation front. The growth of the width of the saturation front is characterized by an apparent dispersion, which we define as half the temporal rate of change of the second centered moment as

$$
D^{a}(t)=\frac{1}{2} \frac{\mathrm{d} \kappa_{11}}{\mathrm{~d} t}
$$

Equations for the moments (39) and thus for $D^{a}(t)$ are derived in Appendix B by invoking first order perturbation theory.

We identify three contributions to $D^{a}(t)$, i.e.

$$
D^{a}(t)=D^{h}(t)+D^{A}(t)+D^{e}(t) .
$$

$D^{h}(t)$ is the contribution to spreading that occurs with the rarefaction wave of the homogeneous solution. $D^{A}(t)$ are the contributions that occur to the nonlocal advection kernel $\mathcal{A}$ and $D^{e}(t)$ those that occur due to the nonlocal dispersive kernels $\mathcal{D}^{(g)}$ and $\mathcal{D}^{(f)}$.

\subsection{Homogeneous contribution to spreading}

The homogeneous contribution $D^{h}(t)$ is given by

$$
D^{h}(t)=\int \mathrm{d} x_{1}\left\{f\left[S_{h}\left(x_{1} / t\right)\right]+\bar{N} g\left[S_{h}\left(x_{1} / t\right)\right]\right\}-t .
$$

The width of the saturation profile evolves purely due to advective widening as expressed by the terms $D^{h}(t)$ and $D^{A}(t)$ and due to actual front spreading as expressed by $D^{e}(t)$. D R A F T September 2, 2010, 4:40pm 


$$
D^{h}(t)=t\left\{\int \mathrm{d} \eta\left\{f\left[S_{h}(\eta)\right]+\bar{N} g\left[S_{h}(\eta)\right]\right\}-1\right\}
$$

Thus, as detailed in [e.g., Bolster et al., 2009a], purely advective effects due to different characteristic velocities lead to a linear evolution of the width of the saturation distribution. Here we observe that for a heavier fluid displacing a lighter one, that is, $\bar{N}<0,(25)$, the increase of the width is slowed down by gravity.

\subsection{Contributions from advective kernels to spreading}

where we defined

$$
\sigma_{k q}^{2}(\eta t)=C_{0}^{k q}(\eta t, \eta t), \quad \sigma_{k q}^{2}(\eta t) l_{k q}(\eta t)=\int_{0}^{\infty} \mathrm{d} x C_{0}^{k q}(\eta t, x) .
$$

$C_{0}^{k q}(\eta t, x)$ is defined in (B5). The variance and correlation length of the permeability field are given by (23). They are constant as $k(\mathbf{x})$ is modeled as a stationary random field. 
Here we identify two contributions, one that evolves linearly with time and a second contribution that evolves towards a constant value at large times.

\subsection{Contributions from dispersive kernels to spreading}

For the contribution $D^{e}(t)$, we obtain in Appendix B

$$
\begin{aligned}
D^{e}(t) & =-\bar{N} \int_{0}^{\infty} \mathrm{d} \eta \frac{\mathrm{d} f\left[S_{h}(\eta)\right]}{\mathrm{d} S_{h}} \frac{\partial g\left[S_{h}(\eta)\right]}{\partial \eta} \eta^{-1} \sigma_{k q}^{2}(\eta t) l_{k q}(\eta t) \\
& -\bar{N}^{2} \int_{0}^{\infty} \mathrm{d} \eta \frac{\mathrm{d} g\left[S_{h}(\eta)\right]}{\mathrm{d} S_{h}} \frac{\partial g\left[S_{h}(\eta)\right]}{\partial \eta} \eta^{-1} \sigma_{k k}^{2}(\eta t) l_{k k}(\eta t) \\
& -\bar{N} \int_{0}^{\infty} \mathrm{d} \eta \frac{\mathrm{d} g\left[S_{h}(\eta)\right]}{\mathrm{d} S_{h}} \frac{\partial f\left[S_{h}(\eta)\right]}{\partial \eta} \eta^{-1} \sigma_{k q}^{2}(\eta t) l_{k q}(\eta t) \\
& -\int_{0}^{\infty} \mathrm{d} \eta \frac{\mathrm{d} f\left[S_{h}(\eta)\right]}{\mathrm{d} S_{h}} \frac{\partial f\left[S_{h}(\eta)\right]}{\partial \eta} \eta^{-1} \sigma_{q q}^{2}(\eta t) l_{q q}(\eta t) .
\end{aligned}
$$

The variance and correlation length of the velocity fluctuations are defined as

$$
\sigma_{q q}^{2}(\eta t)=C_{0}^{q q}(\eta t, \eta t), \quad \sigma_{q q}^{2}(\eta t) l_{q q}(\eta t)=\int_{0}^{\infty} \mathrm{d} x C_{0}^{q q}(\eta t, x) .
$$

$C_{0}^{q q}(\eta t, x)$ is defined in (B5).

\subsection{Approximate solutions of the apparent dispersion coefficients}

In order to further evaluate $D^{A}(t)$ and $D^{e}(t)$ we introduce another approximation (which we justify a posteriori by comparing the numerical and analytical values). For the case of the homogeneous Buckley-Leverett flow it is well known that behind the saturation front the derivative of the fractional flow function $\phi\left(S_{h}\right)$ is given by

$$
\frac{\mathrm{d} \phi\left(S_{h}\right)}{\mathrm{d} S_{h}}=\frac{x_{1}}{t}
$$

at the rear of the saturation profile, see (16). It is this property which allowed Neuweiler et al. [2003] and Bolster et al. [2009a] to evaluate their expressions for the dispersion 
$\mathrm{X}-24$

BOLSTER ET AL.: BUOYANT HETEROGENEOUS MULTIPHASE

coefficients for the non-buoyant case. Buoyancy complicates things in that the fractional flow function is given by the sum of $f(S)$ and $N g(S)$, see (12). Under these conditions, it is no longer trivial to calculate $\frac{\mathrm{d} f(S)}{\mathrm{d} S}$ and $\frac{\mathrm{d} g(S)}{\mathrm{d} S}$. However, we do know their values both at the front as well as the injection boundary. Motivated by the results that emerge from Neuweiler et al. [2003] and Bolster et al. [2009a] we assume that these vary linearly between these two points, that is

$$
\frac{\mathrm{d} f\left(S_{h}\right)}{\mathrm{d} S_{h}}=a_{f} \frac{x_{1}}{t}, \quad \frac{\mathrm{d} g\left(S_{h}\right)}{\mathrm{d} S_{h}}=a_{g} \frac{x_{1}}{t},
$$

for $x_{1}<Q_{f} t$. The constants $a_{f}$ and $a_{g}$ are the respective slopes of $\frac{\mathrm{d} f\left(S_{h}\right)}{\mathrm{d} S_{h}}$ and $\frac{\mathrm{d} g\left(S_{h}\right)}{\mathrm{d} S_{h}}$. These are given by calculating the saturation at the front $S_{h}^{f}$ from condition (15) and substituting it into the respective expression for $\frac{\mathrm{d} f\left(S_{h}^{f}\right)}{\mathrm{d} S_{h}}$ and $\frac{\mathrm{d} g\left(S_{h}^{f}\right)}{\mathrm{d} S}$ for the specific form of relative permeability chosen. A quick study of these functions reveals that in general they do not vary linearly. However, as they appear inside of an integral it may provide a reasonable approximation for quadrature purposes. The numbers $a_{f}$ and $a_{g}$ are obtained by simple interpolation between the the derivatives of $f\left(S_{h}\right)$ and $g\left(S_{h}\right)$ at the front and at the injection point. Note that $a_{f}$ is positive while $a_{g}$ can be positive or negative. The quality of this approximation (50) is discussed Appendix C.

Furthermore we assume that the variances and correlation length in (46) and (48) are constant, which is a reasonable assumption away from the front [e.g., Neuweiler et al., 2003]. Using these approximations and the fact that $S_{h}$ is given by (13), that is $S_{h}^{r}$ is zero 
400

for $x_{1} \geq Q^{f} t, D^{A}(t)$, is given by

$$
\begin{aligned}
D^{A}(t) & =-t\left(\bar{N} \sigma_{k q}^{2} a_{f}+\bar{N}^{2} \sigma_{k k}^{2} a_{g}\right) \int_{0}^{1 / Q^{f}} \mathrm{~d} \eta g\left[S_{h}^{r}(\eta)\right] \\
& +\left(\bar{N} \sigma_{k q}^{2} l_{k q} a_{f}+\bar{N}^{2} \sigma_{k k}^{2} l_{k k} a_{g}\right) \int_{0}^{1 / Q^{f}} \mathrm{~d} \eta \frac{g\left[S_{h}^{r}(\eta)\right]}{\eta} .
\end{aligned}
$$

Note that due to the negative sign in front of the first term, this contribution can lead to a reduction of the linear growth of the saturation distribution. For certain values of the variance and the gravity number it could lead to negative values for the evolution of the front width, which is clearly unphysical. This, however, is a relic of low order perturbation theory.

For the contribution $D^{e}(t)$ these approximations yield

$$
D^{e}(t)=\bar{N} \sigma_{k q}^{2} l_{k q} a_{g}+a_{f} \sigma_{q q}^{2} l_{q q},
$$

where we used that $g\left[S_{h}^{r}(\eta)\right]$ is zero at the injection boundary and at the front, $g\left[S_{h}^{r}(0)\right]=$ $g\left[S_{h}^{r}\left(1 / Q^{f}\right)\right]=0$ and that $f\left[S_{h}^{r}(\eta)\right]$ is one at the injection boundary and zero at the front, $f\left[S_{h}^{r}(0)\right]=1$ and $f\left[S_{h}^{r}\left(1 / Q^{f}\right)\right]=0$. Note that strictly speaking, all the results are only valid for small variances of permeability and velocity.

\subsection{Apparent Dispersion}

西

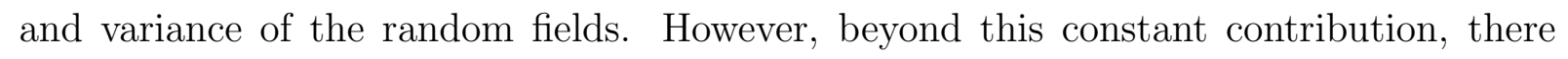


is a further contribution that grows linearly in time given by the first terms in (51). Interestingly, this contribution is independent of the correlation length (a result which we test with numerical simulations in the following section).

The linearity with the correlation length of the constant contributions is in direct analogy to the effective dispersion coefficient in a solute transport problem, which is identical to the macrodispersion coefficient [e.g., Gelhar and Axness, 1983]. The terms that are only proportional to the variance and independent of the correlation length could be interpreted as analogous to an effective permeability in a single phase flow problem, which is also only proportional to the variance and not to the correlation length. The terms proportional to the correlation length can thus be related to an effective dispersion term in the averaged flow equation (36), while the other terms can be related to effective contributions to the gravity term.

The contribution that is linear in time in (51) can thus be interpreted as the way that heterogeneity adds contributions to the buoyant counter-flow of the fluids. This shows that the mean gravity number is only a rough measure to estimate the true flow behavior and does not capture this additional influence of heterogeneity.

\section{Numerical Simulations}

In order to test the solutions presented here we also conducted a numerical study of the buoyant Buckley-Leverett problem in a heterogeneous medium. To do this we used an in house finite volume code, which uses an IPES (Implicit in Pressure and Explicit in Saturation) scheme. The details of the algorithm used can be found in Hasle et al. [2007] and the setup is the same as that used in Bolster et al. [2009a]. The numerical dispersion using this method was generally found to be small compared with the apparent dispersion 
438

$(<10 \%$ typically)we calculate. For situations where buoyancy is excessively stabilizing the condition could not be met.

For each set of parameters 100 random permeability fields were generated using a random generator, which is based on a Fourier transform method. Spatially isotropic permeability fields were generated with a Gaussian distribution, characterized by a relative variance of $\sigma_{k k}^{2}$ and a correlation length of $l_{k k}$. All simulations were performed using square functions as relative permeability functions, i.e.

$$
k_{r i}(S)=S^{2}, \quad k_{r d}(S)=(1-S)^{2} .
$$

Figure 1 shows three sample saturation fields from single realizations using this methodology. The first corresponds to the case where there is no density difference between the two phases, the second where the injected phase is denser and the third where the injected phase is less dense than the displaced one. This figure clearly illustrates the stabilizing and destabilizing effect that buoyancy has on spreading by heterogeneity.

Figure 4 shows the temporal evolution of average saturation profiles (averaged over 100 realizations in each case) for three different cases, clearly displaying the dispersive effect that occurs due to heterogeneity. All cases in this figure are stable. However the influence of buoyancy is evident. The case in the middle where the injected phase is very dense leads to much less spreading than the other two cases. As the system becomes less stabilizing the spreading effect becomes more pronounced. In this work we do not present the results of unstable simulations as it is well known that a perturbation approach such as the one developed here can not capture unstable effects [e.g. Bolster et al., 2009a]. Instead we 
refer the interested reader to works that explore these instabilities [e.g. Riaz and Tchelepi, 2004, 2007; Tartakovsky, 2010].

Figures 5 and 6 illustrate a typical measurement of the dispersion coefficient attributed to heterogeneity. In Figure 5 we illustrate the terms $D^{h}(t)(44)$ for the homogeneous medium and the apparent dispersion coefficient $D^{a}(t)$ (41). The heterogeneity-induced contributions $D^{A}(t) \quad(45)$, and $D^{e}(t),(47)$. are given by the difference of these two lines, which is shown in Figure 6. Note that as predicted by the theory, we have a constant contribution and a contribution that grows linearly in time. To calculate the constant contribution as well as the one that grows linearly in time we perform a best fit of the late time data. The intercept provides the constant contribution, while the slope gives the linear component. The results shown in Figure 6 are normalized by the constant contribution.

\subsection{Influence of Variances}

As mentioned briefly in the previous section the apparent dispersion coefficient in (52) and (51) illustrates various interesting features. For one, it depends proportionally on the variances of the permeability and velocity fields. This suggests that an increase in the variance of the permeability field should lead to a proportional increase in the dispersion coefficient. This means that the constant contribution should be proportionally larger as should the slope of the linear in time contribution (cf. figure 6).

Figure 7 illustrates the normalized dispersion coefficient for a sample case with three different variances, namely $\sigma_{k k}^{2}=0.1,0.5$ and 1 . The dispersion coefficients are normalized by the constant value associated with the $\sigma_{k k}^{2}=0.1$ case (i.e. where the fitting line intersects the vertical axis). As is clearly visible the $\sigma_{k k}^{2}=0.5$ and $\sigma_{k k}^{2}=1$ cases have 
from the figure that the mean front location will lie further ahead of the front associated with this case. This is merely a reflection of the fact that the front location does not scale linearly with gravity number. Thus in a system such as the one we consider here where an array of permeabilities exist it is to be expected that the spreading occurs around a front ahead of that associated with the mean permeability. The effective advection terms are merely telling us that the effective permeability of the system and the mean permeability are not one and the same. Note that the same statement would hold if we had expanded the intrinsic permeability around the geometric mean. Panilov and Floriat [2004], who studied a similar problem using homogenization, also found that the mean and effective permeability are not the same. However, they claimed that they only expect the two to be different for nonstationary random permeability fields. In this work our fields can be stationary and we still find a discrepancy. The effective advection term could also lead to an effective shape of the gravity function, so that the introduction of an effective permeability would not be sufficient.

\section{Conclusions}

(1)

In the introduction to this article we posed a series of questions regarding the influence of buoyancy and heterogeneity on spreading in two phase flow under the Buckley-Leverett approximation. We remind the reader that these were

1. Can we, using perturbation theory, asses the rate of spreading that occurs?

2. What measures of the heterogeneous field (e.g. variance, correlation length) control this spreading? Also, why and how do they? 
3. What influence does the heterogeneity in gravity number have? And does the arithmetic mean of the gravity number represent a mean behavior in the heterogeneous system?

The answer to the first question is that following the methodology of Neuweiler et al. [2003] and Bolster et al. [2009a], where perturbation theory around the mean behavior is employed, we can estimate the apparent dispersion coefficient, which is a measure for the spreading of the front. The dispersion coefficient that arises is more complex than for the case without buoyancy. When we write an effective equation there are now six distinct nonlocal terms that contribute to it. Four of these terms have the appearance of an effective dispersion and the first of these terms is identical to the case without buoyancy. The other two additional terms look more like contributing as effective advections. This is distinctly different from the case with no buoyancy.

The answers to the second and the third question are closely related. We explored the different contributions to the front spreading and illustrate that only two of the dispersive nonlocal terms seem to play an important role in spreading of the interface. These terms are proportional to the variance and the correlation length of the heterogeneous fields. The terms that are advective in appearance appear to have no influence on the actual spreading of the front. Instead these terms reflect the location of the front around which spreading occurs. It is proportional to the variance of the heterogeneous fields, but not related to the correlation length. This front is typically further ahead of the front obtained in a homogeneous field with the arithmetic mean of the intrinsic permeability. Thus these terms represent an effective contribution to the gravity term, which might be an effective intrinsic permeability different from the arithmetic mean. This is unexpected according to previous works. As stabilization slows the front down and leads to a more 
compact saturation profile, the influence of heterogeneity combined with buoyancy is a diminishing of the stabilization effect on the averaged front. This effect is not captured by the arithmetic average of the gravity number. The arithmetic mean of the gravity number does thus not capture the whole flow behavior in a heterogeneous field.

Finally, it is remarkable that the time behavior of the different contributions to the apparent dispersion could be confirmed by numerical simulations even in a quantitative manner, although they are derived from applying linear perturbation theory to a highly non-linear problem. When carrying out numerical simulations in fields with large variances, this is no longer true and demonstrates the limitations of the perturbation approximation used here.

\section{Appendix A: Green Function}

The Green function for a homogeneous medium satisfies the equation

$$
\frac{\partial G_{0}\left(x_{1}, t \mid x_{1}^{\prime}, t^{\prime}\right)}{\partial t}+\frac{\partial}{\partial x_{1}} \frac{\mathrm{d} \phi\left[S_{h}\left(x_{1}, t\right)\right]}{\mathrm{d} S_{h}} G_{0}\left(x_{1}, t \mid x_{1}^{\prime}, t^{\prime}\right)=0
$$

for the initial condition $G_{0}\left(x_{1}, t^{\prime} \mid x_{1}^{\prime}, t^{\prime},\right)=\delta\left(x_{1}-x_{1}^{\prime}\right)$. Analyzing the homogeneous problem (11) using the method of characteristics [e.g., Marle, 1981], one finds that the derivative of the total flow function $\phi\left[S_{h}\left(x_{1}, t\right)\right]$ with respect to $S_{h}$ is the velocity of the characteristic of $S_{h}\left(x_{1}, t\right)$ at $x_{1}$ at time $t$. The fact the characteristic velocity for a given saturation is constant, means that the saturation at a given point was transported there by a constant velocity, which is given by

$$
\frac{\mathrm{d} \phi\left[S_{h}\left(x_{1}, t\right)\right]}{\mathrm{d} S}=\frac{x_{1}}{t}
$$

This simplifies (A1) to

$$
\frac{\partial G_{0}\left(x_{1}, t \mid x_{1}^{\prime}, t^{\prime}\right)}{\partial t}+\frac{1}{t} \frac{\partial}{\partial x_{1}} x_{1} G_{0}\left(x_{1}, t \mid x_{1}^{\prime}, t^{\prime}\right)=0 .
$$


607

The latter can be solved by the method of characteristics and gives

$$
G_{0}\left(x_{1}, t \mid x_{1}^{\prime}, t^{\prime}\right)=\frac{1}{t} \delta\left(\frac{x_{1}^{\prime}}{t^{\prime}}-\frac{x_{1}}{t}\right)
$$

608

\section{Appendix B: Spatial Moment Equations and Apparent Dispersion}

Applying definition (38) to (36) we obtain an equation for $\bar{s}(\mathbf{x}, t)$

$$
\begin{aligned}
& \frac{\partial \bar{s}(\mathbf{x}, t)}{\partial t}=L^{-1} \frac{\partial^{2} f[\bar{S}(\mathbf{x}, t)]}{\partial x_{1}^{2}}+L^{-1} \bar{N} \frac{\partial^{2} g[\bar{S}(\mathbf{x}, t)]}{\partial x_{1}^{2}} \\
& -L^{-1} \frac{\partial}{\partial x_{1}} \nabla \cdot \int \mathrm{d} \mathbf{x}^{\prime} \int_{0}^{t} \mathrm{~d} t^{\prime} \mathcal{A}\left(\mathbf{x}, t \mid \mathbf{x}^{\prime}, t^{\prime}\right) g\left[\bar{S}\left(\mathbf{x}^{\prime}, t^{\prime}\right)\right] \\
& -L^{-1} \frac{\partial}{\partial x_{1}} \nabla \cdot \int \mathrm{d} \mathbf{x}^{\prime} \int_{0}^{t} \mathrm{~d} t^{\prime} \mathcal{D}^{(g)}\left(\mathbf{x}, t \mid \mathbf{x}^{\prime}, t^{\prime}\right) \nabla^{\prime} g\left[\bar{S}\left(\mathbf{x}^{\prime}, t^{\prime}\right)\right] \\
& -L^{-1} \frac{\partial}{\partial x_{1}} \nabla \cdot \int \mathrm{d} \mathbf{x}^{\prime} \int_{0}^{t} \mathrm{~d} t^{\prime} \mathcal{D}^{(f)}\left(\mathbf{x}, t \mid \mathbf{x}^{\prime}, t^{\prime}\right) \nabla^{\prime} f\left[\bar{S}\left(\mathbf{x}^{\prime}, t^{\prime}\right)\right]
\end{aligned}
$$



we define

$$
\varphi_{f}\left(x_{1} / t\right)=f\left[S_{h}\left(x_{1} / t\right] .\right.
$$

using the fact that $S_{h}$ has the scaling form (13). Furthermore, we define the advection kernel $\mathcal{A}_{h}\left(x_{1}, t \mid x_{1}^{\prime}, t^{\prime}\right)$ by

$$
\begin{aligned}
\mathcal{A}_{h}\left(x_{1}, t \mid x_{1}^{\prime}, t^{\prime}\right) & =\bar{N} \phi_{f}\left(x_{1} / t\right) \frac{1}{t} \delta\left(\frac{x_{1}}{t}-\frac{x_{1}^{\prime}}{t^{\prime}}\right) \frac{\partial C_{0}^{k q}\left(x_{1}, x_{1}^{\prime}\right)}{\partial x_{1}^{\prime}} \\
& +\bar{N} \phi_{g}\left(x_{1} / t\right) \frac{1}{t} \delta\left(\frac{x_{1}}{t}-\frac{x_{1}^{\prime}}{t^{\prime}}\right) \frac{\partial C_{0}^{k k}\left(x_{1}-x_{1}^{\prime}\right)}{\partial x_{1}^{\prime}} .
\end{aligned}
$$

where we used the explicit form (A4) of the homogeneous Green function. Additionally,

$$
\phi_{f}\left(x_{1} / t\right)=\frac{\mathrm{d} f\left[S_{h}\left(x_{1} / t\right)\right]}{\mathrm{d} S_{h}}, \quad \quad \phi_{g}\left(x_{1} / t\right)=\frac{\mathrm{d} g\left[S_{h}\left(x_{1} / t\right)\right]}{\mathrm{d} S_{h}}
$$


622 623

using again the fact that $S_{h}$ has the scaling form (13). With all this, the dispersion kernels are given by

$$
\begin{aligned}
\mathcal{D}_{h}^{(g)}\left(x_{1}, t \mid x_{1}^{\prime}, t^{\prime}\right) & =\bar{N} \phi_{g}\left(x_{1} / t\right) \frac{1}{t} \delta\left(\frac{x_{1}}{t}-\frac{x_{1}^{\prime}}{t^{\prime}}\right) C_{0}^{k q}\left(x_{1}, x_{1}^{\prime}\right) \\
& +\bar{N}^{2} \phi_{g}\left(x_{1} / t\right) \frac{1}{t} \delta\left(\frac{x_{1}}{t}-\frac{x_{1}^{\prime}}{t^{\prime}}\right) C_{0}^{k k}\left(x_{1}-x_{1}^{\prime}\right) . \\
\mathcal{D}_{h}^{(f)}\left(x_{1}, t \mid x_{1}^{\prime}, t^{\prime}\right) & =\bar{N} \phi_{f}\left(x_{1} / t\right) \frac{1}{t} \delta\left(\frac{x_{1}}{t}-\frac{x_{1}^{\prime}}{t^{\prime}}\right) C_{0}^{q q}\left(x_{1}, x_{1}^{\prime}\right) \\
& +\phi_{f}\left(x_{1} / t\right) \frac{1}{t} \delta\left(\frac{x_{1}}{t}-\frac{x_{1}^{\prime}}{t^{\prime}}\right) C_{0}^{k q}\left(x_{1}, x_{1}^{\prime}\right),
\end{aligned}
$$

where we define the correlation function as

$$
C_{0}^{k q}\left(x_{1}, x_{1}^{\prime}\right)=\left.C_{1}^{k q}\left(\mathbf{x}, \mathbf{x}^{\prime}\right)\right|_{x_{2}=x_{2}^{\prime}=0} .
$$

${ }_{25} \quad C_{0}^{q q}\left(x_{1}, x_{1}^{\prime}\right)$ and $C_{0}^{k k}\left(x_{1}, x_{1}^{\prime}\right)$ are defined correspondingly.

We obtain an expression for the time derivative of $m_{1}^{(1)}(t)$ by multiplying (B2) by $x_{1}$ and subsequent integration over space. This gives

$$
\frac{\mathrm{d} m_{1}^{(1)}(t)}{\mathrm{d} t}=1
$$

where we used that $S_{h}(0, t)=1$ and the fact that $f(1)=1, f(0)=0, g(0)=g(1)=0$, and that $\mathcal{A}_{h}\left(x_{1}, t \mid x_{1}^{\prime}, t^{\prime}\right)$ is zero at $x_{1}=0$ and $x_{1}=\infty$. The evolution equation of the second moment $m_{11}^{(2)}(t)$ is obtained by multiplying (B2) by $x_{1}$ and subseqent integration 
over space

$$
\begin{aligned}
\frac{\mathrm{d} m_{11}^{(2)}(t)}{\mathrm{d} t} & =2 \int \mathrm{d} x_{1} \phi\left[S_{h}\left(x_{1} / t\right)\right] \\
& -2 \int \mathrm{d} x_{1} \int \mathrm{d} x_{1}^{\prime} \int_{0}^{t} \mathrm{~d} t^{\prime} \mathcal{A}_{h}\left(x_{1}, t \mid x_{1}^{\prime}, t^{\prime}\right) \varphi_{g}\left(x_{1}^{\prime} / t^{\prime}\right) \\
& -2 \int \mathrm{d} x_{1} \int \mathrm{d} x_{1}^{\prime} \int_{0}^{t} \mathrm{~d} t^{\prime} \mathcal{D}_{h}^{(g)}\left(x_{1}, t \mid x_{1}^{\prime}, t^{\prime}\right) \frac{\left.\partial \varphi_{g}\left(x_{1}^{\prime} / t^{\prime}\right)\right]}{\partial x_{1}^{\prime}} \\
& -2 \int \mathrm{d} x_{1} \int \mathrm{d} x_{1}^{\prime} \int_{0}^{t} \mathrm{~d} t^{\prime} \mathcal{D}_{h}^{(f)}\left(x_{1}, t \mid x_{1}^{\prime}, t^{\prime}\right) \frac{\partial \varphi_{f}\left(x_{1}^{\prime} / t^{\prime}\right)}{\partial x_{1}^{\prime}} .
\end{aligned}
$$

${ }_{632}$ Note that the apparent dispersion coefficient (41) is expressed in terms of $m_{1}^{(1)}(t)$ and 633 $m_{11}^{(2)}(t)$ as

$$
D^{a}(t)=\frac{1}{2} \frac{\mathrm{d} m_{11}^{(2)}}{\mathrm{d} t}-m_{1}^{(1)}(t) \frac{\mathrm{d} m_{1}^{(1)}}{\mathrm{d} t}
$$

Therefore, combining (B6) and (B7), $D^{a}(t)$ can be decomposed as in (42) with

$$
\begin{aligned}
D^{h}(t) & =\int \mathrm{d} x_{1} \phi\left[S_{h}\left(x_{1} / t\right)\right]-t \\
D^{A}(t) & =-\int \mathrm{d} x_{1} \int \mathrm{d} x_{1}^{\prime} \int_{0}^{t} \mathrm{~d} t^{\prime} \mathcal{A}_{h}\left(x_{1}, t \mid x_{1}^{\prime}, t^{\prime}\right) \varphi_{g}\left(x_{1}^{\prime} / t^{\prime}\right) \\
D^{e}(t) & =-\int \mathrm{d} x_{1} \int \mathrm{d} x_{1}^{\prime} \int_{0}^{t} \mathrm{~d} t^{\prime} \mathcal{D}_{h}^{(g)}\left(x_{1}, t \mid x_{1}^{\prime}, t^{\prime}\right) \frac{\left.\partial \varphi_{g}\left(x_{1}^{\prime} / t^{\prime}\right)\right]}{\partial x_{1}^{\prime}} \\
& -\int \mathrm{d} x_{1} \int \mathrm{d} x_{1}^{\prime} \int_{0}^{t} \mathrm{~d} t^{\prime} \mathcal{D}_{h}^{(f)}\left(x_{1}, t \mid x_{1}^{\prime}, t^{\prime}\right) \frac{\partial \varphi_{f}\left(x_{1}^{\prime} / t^{\prime}\right)}{\partial x_{1}^{\prime}} .
\end{aligned}
$$

${ }_{635}$ Inserting the kernel $\mathcal{A}_{h}(t)$ defined by (B4a), we notice that $D^{A}(t)$, can be written as

$$
D^{A}(t)=\bar{N} M^{A}\left(\left\{\phi_{f}\right\},\left\{C_{0}^{k q}\right\},\left\{\varphi_{g}\right\}, t\right)+\bar{N}^{2} M^{A}\left(\left\{\phi_{g}\right\},\left\{C_{0}^{k k}\right\},\left\{\varphi_{g}\right\}, t\right)
$$


636

where the functional $M^{A}(\{\phi\},\{C\},\{\varphi\}, t)$ is defined by

$$
\begin{aligned}
& M^{A}(\{\phi\},\{C\},\{\varphi\}, t)= \\
& -\int_{0}^{\infty} \mathrm{d} x_{1} \int_{0}^{t} \mathrm{~d} t^{\prime} \int_{0}^{\infty} \mathrm{d} x_{1}^{\prime} \phi\left(\frac{x_{1}}{t}\right) \frac{1}{t} \delta\left(\frac{x_{1}}{t}-\frac{x_{1}^{\prime}}{t^{\prime}}\right) \frac{\partial C\left(x_{1}, x_{1}^{\prime}\right)}{\partial x_{1}^{\prime}} \varphi\left(x_{1}^{\prime} / t^{\prime}\right),
\end{aligned}
$$

${ }_{637} \quad$ We now rescale $x_{1}=\eta t$ and $x_{1}^{\prime}=\eta^{\prime} t^{\prime}$. This gives

$$
\begin{aligned}
& M^{A}(\{\phi\},\{C\},\{\varphi\}, t)= \\
& -\int_{0}^{\infty} \mathrm{d} \eta \int_{0}^{t} \mathrm{~d} t^{\prime} \int_{0}^{\infty} \mathrm{d} \eta^{\prime} \phi(\eta) \delta\left(\eta-\eta^{\prime}\right) t^{\prime} C^{\prime}\left(\eta t, \eta^{\prime} t^{\prime}\right) \varphi\left(\eta^{\prime}\right),
\end{aligned}
$$

${ }^{638}$ where $C^{\prime}(a, x)=\frac{\partial C(a, x)}{\partial x}$. Executing the $\eta^{\prime}$-integration gives

$$
M^{A}(\{\phi\},\{C\},\{\varphi\}, t)=-\int_{0}^{\infty} \mathrm{d} \eta \int_{0}^{t} \mathrm{~d} t^{\prime} \phi(\eta) t^{\prime} C^{\prime}\left(\eta t, \eta t^{\prime}\right) \varphi(\eta) .
$$

639 Rescaling time as $t^{\prime}=x / \eta$, we obtain

$$
M^{A}(\{\phi\},\{C\},\{\varphi\}, t)=-\int_{0}^{\infty} \mathrm{d} \eta \phi(\eta) \varphi(\eta) \eta^{-2} \int_{0}^{t \eta} \mathrm{d} x x C^{\prime}(\eta t, x) .
$$

640

Integration by parts gives

$$
\begin{aligned}
& M^{A}(\{\phi\},\{C\},\{\varphi\}, t)= \\
& -\int_{0}^{\infty} \mathrm{d} \eta \phi(\eta) \varphi(\eta)\left[\eta^{-1} t C(\eta t, \eta t)+\eta^{-2} \int_{0}^{t \eta} \mathrm{d} x C(\eta t, x)\right] .
\end{aligned}
$$

${ }_{641}$ For dimensionless times $t \gg 1$, we approximate the latter by

$$
\begin{aligned}
& M^{A}(\{\phi\},\{C\},\{\varphi\}, t)= \\
& -\int_{0}^{\infty} \mathrm{d} \eta \phi(\eta) \varphi(\eta)\left[\eta^{-1} t C(\eta t, \eta t)+\eta^{-2} \int_{0}^{\infty} \mathrm{d} x C(\eta t, x+\eta t)\right] .
\end{aligned}
$$

${ }_{642}$ Similarly, we observe that $D^{e}(t),(B 11)$, can be written in the unified form

$$
\begin{aligned}
D^{e}(t) & =\bar{N} M^{e}\left(\left\{\phi_{f}\right\},\left\{C_{0}^{k q}\right\},\left\{\varphi_{g}\right\}, t\right)+\bar{N}^{2} M^{e}\left(\left\{\phi_{g}\right\},\left\{C_{0}^{k k}\right\},\left\{\varphi_{g}\right\}, t\right) \\
& +\bar{N} M^{e}\left(\left\{\phi_{g}\right\},\left\{C_{0}^{k q}\right\},\left\{\varphi_{f}\right\}, t\right)+M^{e}\left(\left\{\phi_{f}\right\},\left\{C_{0}^{q q}\right\},\left\{\varphi_{f}\right\}, t\right),
\end{aligned}
$$


643

where the functional $M^{e}(\{\phi\},\{C\},\{\varphi\}, t)$ is defined by

$$
\begin{aligned}
& M^{e}(\{\phi\},\{C\},\{\varphi\}, t)= \\
& -\int_{0}^{\infty} \mathrm{d} x_{1} \int_{0}^{t} \mathrm{~d} t^{\prime} \int_{0}^{\infty} \mathrm{d} x_{1}^{\prime} \phi\left(\frac{x_{1}}{t}\right) \frac{1}{t} \delta\left(\frac{x_{1}}{t}-\frac{x_{1}^{\prime}}{t^{\prime}}\right) C\left(x_{1}, x_{1}^{\prime}\right) \frac{\partial \varphi\left(x_{1}^{\prime} / t^{\prime}\right)}{\partial x_{1}^{\prime}} .
\end{aligned}
$$

644

$$
M^{e}(\{\phi\},\{C\},\{\varphi\}, t)=-\int_{0}^{\infty} \mathrm{d} \eta \phi(\eta) \frac{\partial \varphi(\eta)}{\partial \eta} \eta^{-1} \int_{0}^{t \eta} \mathrm{d} x C(\eta t, x) .
$$

As above, we approximate the latter for times $t \gg 1$ by

$$
M^{e}(\{\phi\},\{C\},\{\varphi\}, t)=-\int_{0}^{\infty} \mathrm{d} \eta \phi(\eta) \frac{\partial \varphi(\eta)}{\partial \eta} \eta^{-1} \int_{0}^{\infty} \mathrm{d} x C(\eta t, x+\eta t) .
$$

\section{Appendix C: Integral Approximations}

The approximation (50) considerably reduces the complexity of this problem. To illustrate that this approximation works well we consider the following integrals

$$
\begin{aligned}
& A_{f}=\int_{0}^{\infty} \mathrm{d} x \frac{t}{x} \frac{\mathrm{d} f\left[S_{h}(x / t)\right]}{\mathrm{d} S_{h}} \frac{\mathrm{d} f\left[S_{h}(x / t)\right]}{\mathrm{d} x} \\
& A_{g}=\int_{0}^{\infty} \mathrm{d} x \frac{t}{x} \frac{\mathrm{d} g\left[S_{h}(x / t)\right]}{\mathrm{d} S_{h}} \frac{\mathrm{d} f\left[S_{h}(x / t)\right]}{\mathrm{d} x}
\end{aligned}
$$

Using the approximation (50) we obtain

$$
\begin{aligned}
& A_{f}=-a_{f} \\
& A_{g}=-a_{g}
\end{aligned}
$$

These integrals arise naturally if one were to consider a delta correlated permeability field, which can be thought of as a limit of many other correlation functions. Figure 11 compares the integrals obtained numerically and calculated by using approximation (50). Figure 11a illustrates $A_{f}$. For all values of $N$ and $M$ chosen, the approximation works 
very well. Similarly Figure $11 \mathrm{~b}$ shows the numerical evaluation of $A_{g}$ compared to $a_{f}$. The agreement is very good for larger values of $M$. For small values of $M$ the approximation only seems to work for values of $N$ that are not close to 0 .

\section{References}

Aris, R., On the dispersion of solute in a fluid flow through a tube, Proc Roy Soc A, 235, 67-77, 1956.

Bachu, S., Co2 storage in geological media: Role, means, status and barriers to deployment, Progress in Energy and Combustion Science, 34, 254-273, 2008.

Bachu, S., and J. Adams, Sequestration of co2 in geological media in response to climate change: capacity of deep saline aquifers to sequester co2 in solution, Energy Conversion \& Management, 44, 31513175, 2003.

Bear, J., Dynamics of fluids in porous media, Dover Publications, 1988.

Binning, P., and M. Celia, Practical implementation of the fractional flow approach to multi-phase flow simulation, Advances in Water Resources, 22, 461-478, 1999.

Bolster, D., M. Dentz, and J. Carrera, Effective two phase flow in heterogeneous media under temporal pressure fluctuations, Water Resources Research, In Press, 1-12, 2009a.

Bolster, D., M. Dentz, and T. LeBorgne, Solute dispersion in channels with periodically varying apertures, Physics of Fluids, 21, 056,601, 2009b.

Bolster, D. T., D. M. Tartakovsky, and M. Dentz, Analytical models of contaminant transport in coastal aquifers, Advances in Water Resources, 30, 1962-197, 2007.

Brenner, H., and D. Edwards, Macrotransport Processes, Butterworth-Heinemann, MA, USA, 1993. 
Bryant, S., S. Lakshminarasimhan, and G. Pope, Buoyancy-dominated multiphase flow and its effect on geological sequestration of $\mathrm{co}_{2}$, SPE Journal, 13(4), 447-454, 2008.

Cushman, J. H., X. Hu, and T. R. Ginn, Nonequilibrium statistical mechanics of preasymptotic dispersion, J. Stat. Phys., 75(5/6), 859-878, 1994.

Cvetovic, V., and G. Dagan, Reactive transport and immiscible flow in geological media. ii. applications, Proc. R. Soc. Lond. A, 452, 202-328, 1996.

Dagan, G., Flow and Transport in Porous Formations, Springer, New York, 1989.

Dagan, G., and V. Cvetkovic, Reactive transport and immiscible flow in geological media i general theory, P. Roy. Soc. Lond. A, 452, 285-301, 1996.

Dentz, M., and J. Carrera, Mixing and spreading in stratified flow, Physics of Fluids, 19, 017107, 017,107, 2007.

Dentz, M., D. Tartakovsky, E. Abarca, A. Guadagnini, and X. S.-V. J. Carrera, Variabledensity flow in porous media, Journal of Fluid Mechanics, 561, 209-235, 2006.

Dong, M., S. Ma, and Q. Liu, Enhanced oil recovery through interfacial instability: a study of chemical flooding for brintnell heavy oil, Fuel, 88, 1049-1056, 2009.

Efendiev, Y., and L. Durlofsky, Numerical modeling of subgrid heterogeneity in two phase flow simulations, Water Resour Res, 38, 10.1029/2000WR000,190,, 2002.

Ferguson, R., C. Nichols, T. van Leeusen, and V. Kuuskraa, Storing co2 with enhanced oil recovery, Energy Procedia, 1, 1989-1996, 2009.

Gelhar, L., Stochastic Subsurface Hydrology, Prentice Hall, Englewood Cliffs, NJ, 1993.

Gelhar, L., and C. Axness, Three-dimensional stochastic analysis of macrodispersion in aquifers, Water Resources REsearch, 19, 161, 1983. 
Graf, T., and R. Therrien, A test case for the simulation of three-dimensional variabledensity flow and solute transport in discretely-fractured porous media, Advances in Water Resources, 31, 1352-1363, 2008.

Hasle, G., K. Lie, and E. Quak, Geometric Modelling, Numerical Simulation, and Optimization, Springer Berlin Heidelberg, Germany, 2007.

Henry, H., Effects of dispersion on salt encroachment in coastal aquifers, Water Supply Pap., 1613-C, 1964.

Huppert, H., and A. Woods, Gravity driven flow in porous layers, Journal of Fluid Mechanics, 292, 55-69, 1995.

Kalejaiye, B., and S. Cardoso, Specification of the dispersion coefficient in the modeling of gravity-driven flow in porous media, Water Resour Res 2005;41., 41, W10,407, doi:10.1029/2004WR003,925, 2005.

Kempers, L., and H. Haas, The dispersion zone between fluids with different density and viscosity in a heterogeneous porous mediums, J Fluid Mech, 267, 299-324, 1994.

Kitanidis, P., Prediction by the method of moments of transport in heterogeneous formations, Journal of Hydrology, 102, 453, 1988.

Koch, D. L., and J. F. Brady, A non-local description of advection-diffusion with application to dispersion in porous media, J. Fluid Mech., 180, 387-403, 1987.

Kubo, R., M. Toda, and N. Hashitsume, Statistical Physics II, Non-Equilibrium Statistical Mechanics, Springer Verlag Berlin Heidelberg, 1991.

Lake, L., Enhanced Oil Recovery, Prentice Hill, Englewood Cliffs, New Jersey, 1989.

Langlo, P., and M. Espedal, Macrodispersion for two-phase, immiscible flow in porous media, Advances in Water Resources, 17, 297-316, 1995. 
Marle, C., Multiphase Flow in Porous Media, Institut Francais du Petrole, Paris, 1981.

Neuman, S. P., Eulerian-lagangian theory of transport in space-time nonstationary velocity fields: Exact nonlocal formalism by conditional moments and weak approximation, Water Resour. Res., 29(3), 633-645, 1993.

Neuman, S. P., and D. M. Tartakovsky, Perspective on theories of anomalous transport in heterogeneous media, Adv. Water Resour., 32, 670-680, 2009.

Neuweiler, I., S. Attinger, W. Kinzelbach, and P. King, Large scale mixing for immiscible displacement in heterogenous porous media, Transport in Porous Media, 51, 287-314, 2003.

Neuweiler, I., A. Papafotiou, H. Class, and R. Helmig, Estimation of effective parameters for a two-phase flow problem in non-gaussian heterogeneous porous media, Journal of Contaminant Hydrology, In Press, doi:10.1016/j.jconhyd.2010.08.001, 2010.

Noetinger, B., V. Artus, and L. Ricard, Dynamics of the water-oil front for two-phase, immiscible flow in heterogeneous porous media. 2 isotropic media, Transport in Porous Media, 56, 305-328, 2004.

Panilov, M., and S. Floriat, Nonlinear two-phase mixing in heterogeneous porous media, Transport in Porous Media, 57, 347-375, 2004.

Riaz, A., and H. Tchelepi, Linear stability analysis of immiscible two-phase flow in porous media with capillary dispersion and density variation, Physics of Fluids, 16, 4727 doi:10.1063/1.1812,511, 2004.

Riaz, A., and H. Tchelepi, Numerical simulations of immiscible two-phase flow in porous media, Physics of Fluids, 18, 014,104-014,104-12, 2006. 
Riaz, A., and H. Tchelepi, Stability of two-phase vertical flow in homogeneous porous media, Physics of Fluids, 19, 072,103 doi:10.1063/1.2742,975, 2007.

Riaz, A., and H. Tchelepi, Dynamics of vertical displacement in porous media associated with $\mathrm{CO}_{2}$ sequestration, SPE Journal, 13(3), 305-313, 2008.

Rubin, Y., Applied Stochastic Hydrogeology, Oxford University Press, New York, USA, 2003.

Saffman, P., and G. Taylor, The penetration of a fluid into a porous medium or hele-shaw cell containing a more viscous liquid, Proc. Roy. Soc. A, 245, 312-329, 1958.

Tartakovsky, A. M., Lagrangian simulations of unstable gravity-driven flow of fluids with variable density in randomly heterogeneous porous media, Stoch Environ Res Risk Assess, In Press, DOI 10.1007/s00,477-010-0402-3, 2010.

Tartakovsky, D. M., and S. P. Neuman, Transient flow in bounded randomly heterogeneous domains 1. Exact conditional moment equations and recursive approximations, Water Resour. Res., 34(1), 1-12, 1998.

Tokunaga, T., K. Mogi, O. Matsubara, H. Tosaka, and K. Kojima, Buoyancy and interfacial force effects on two-phase displacement patterns: An experimental study, $A A P G$ Bulletin, 84(1), 65-64, 2000.

Welty, C., and L. Gelhar, Stochastic analysis of the effects of fluid density and viscosity variability on macrodispersion in heterogeneous porous media,, Water Resour. Res., 27 (8), 2061-2075, 1991.

Zhang, D., and H. Tchelepi, Stochastic analysis of immiscible two-phase flow in heterogeneous media, SPE Journal, 4, 380-388, 1999. 
763 1961. 

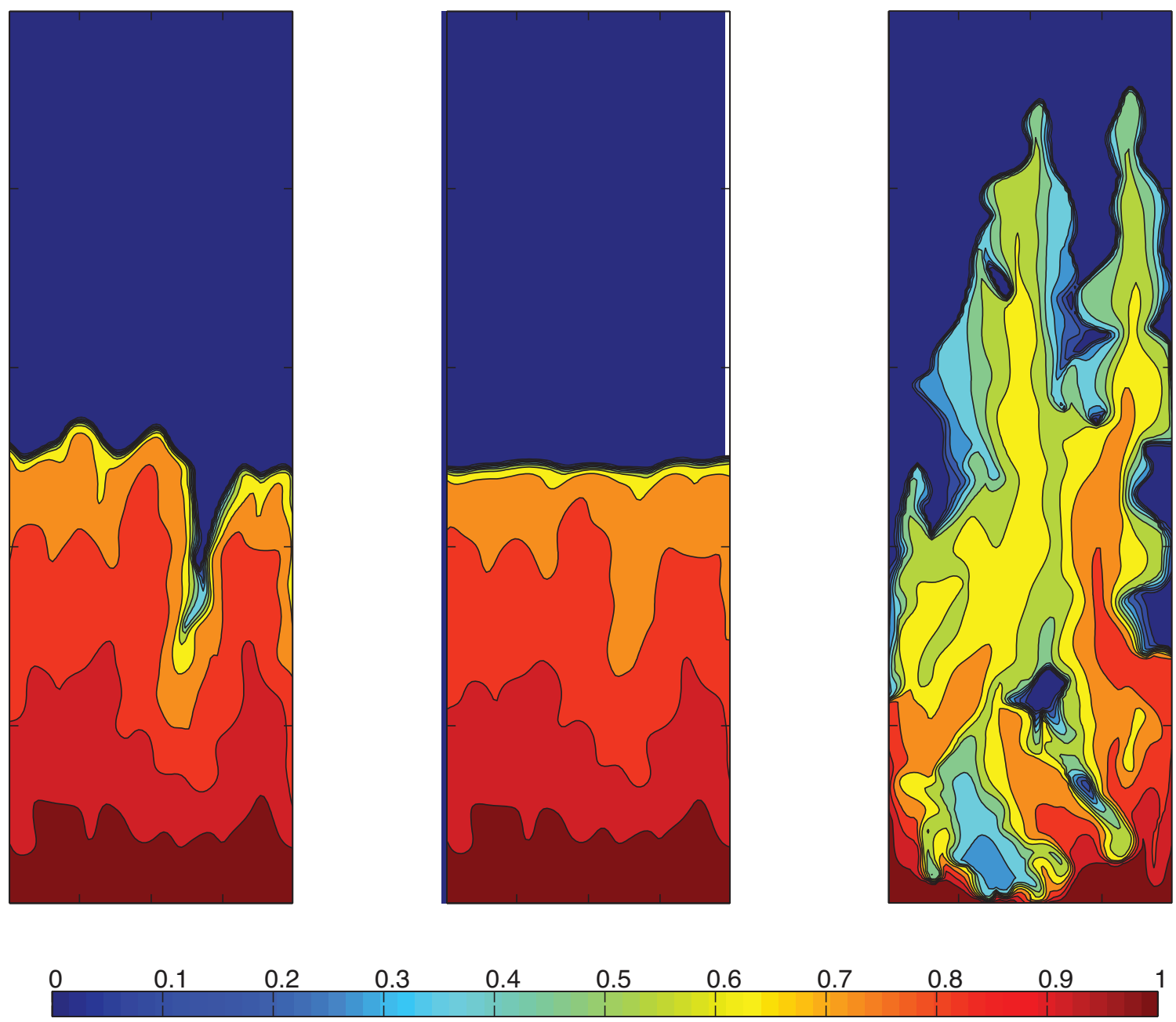

Figure 1. Sample contour plots of saturation within the same random permeability field. In all cases the viscosity ratio $M=1$. (left) Zero Buoyancy, Neutrally Stable (middle) Buoyantly stable case (right) Buoyantly unstable case. The colourbar displays saturations from 0 to 1. 


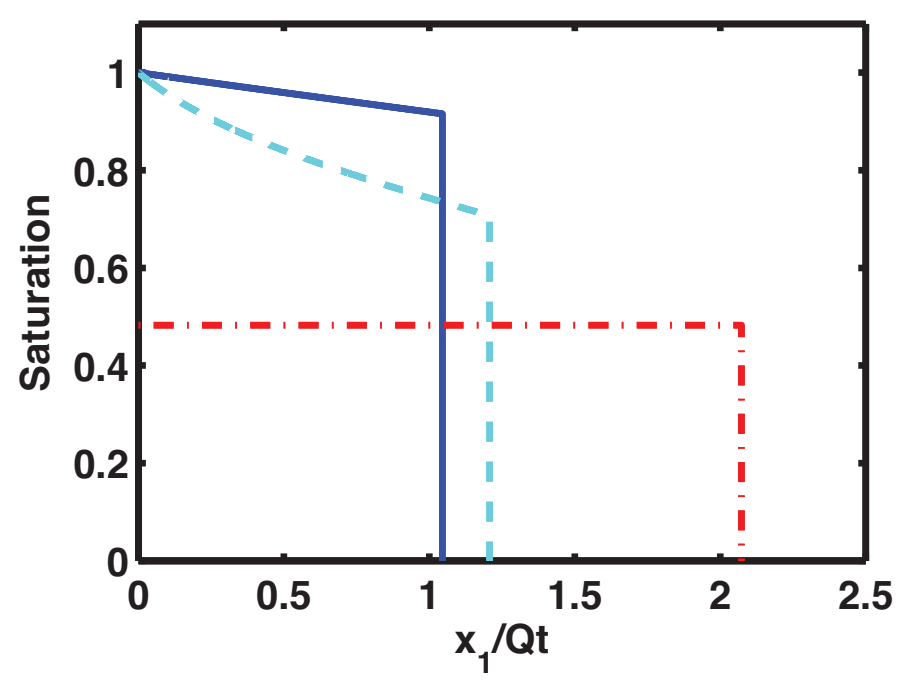

(a)

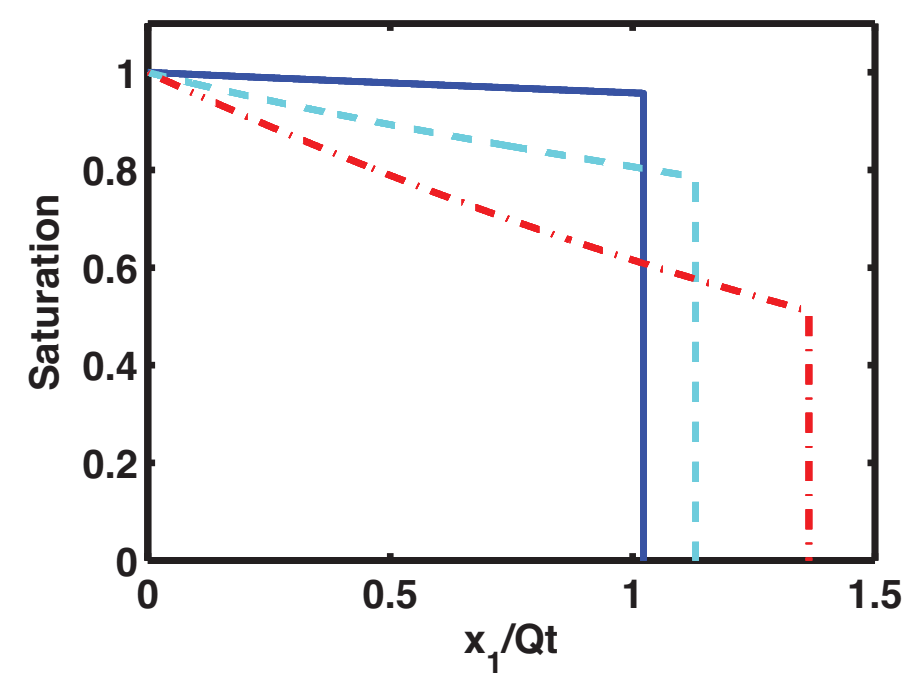

(b)

Figure 2. (a) Normalized homogeneous solution to Buckley-Leverett displacement for $M=1, N=5(-$.), $0(--),-5(-)$ and (b) $N=-1$ and various values of $M . M=0.1$ (red - .), $M=1$ (light blue - -) and $M=10$ ( dark blue -). The front location is normalized by $Q t$ reflecting the self similar in time nature of this solution. 


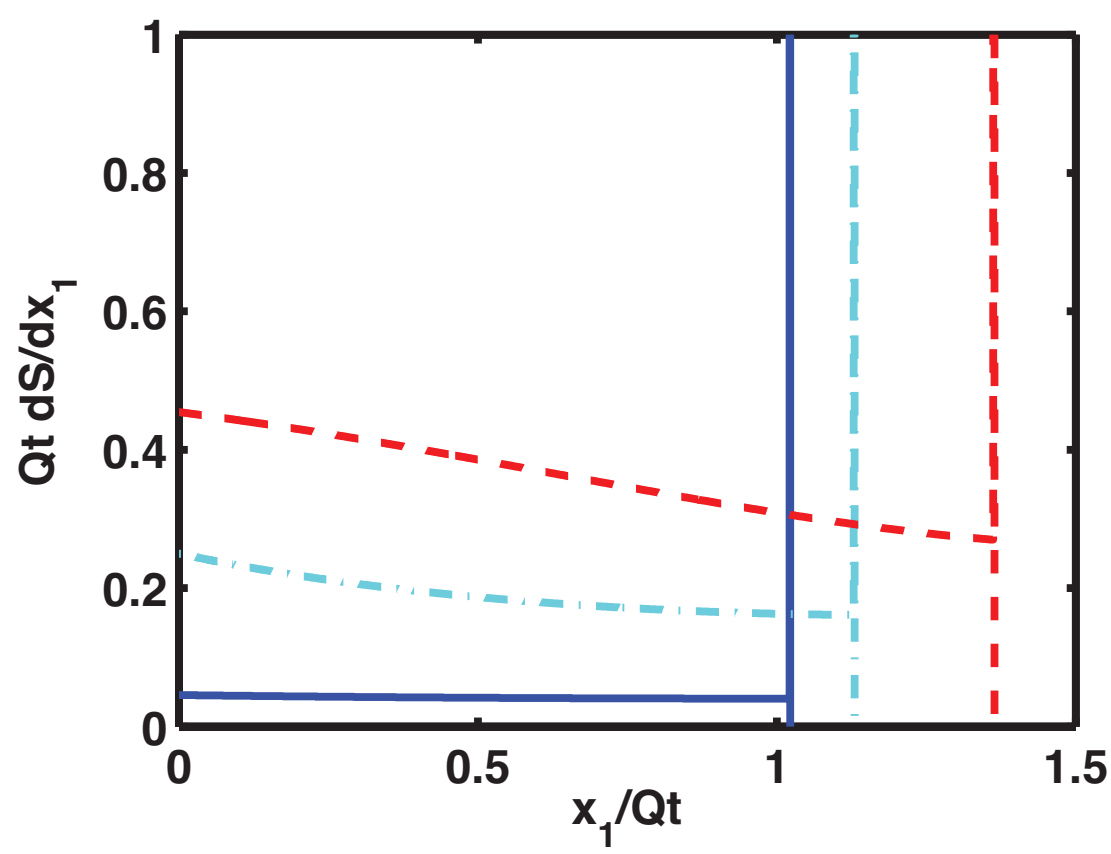

(a)

Figure 3. Normalized derivative of saturation $\frac{d S}{d x_{1}}$ calculated from equation (17) for various values of $M=0.1$ (red - .), 1 (light blue - -) and 10 (black -) and $N=-1$. 

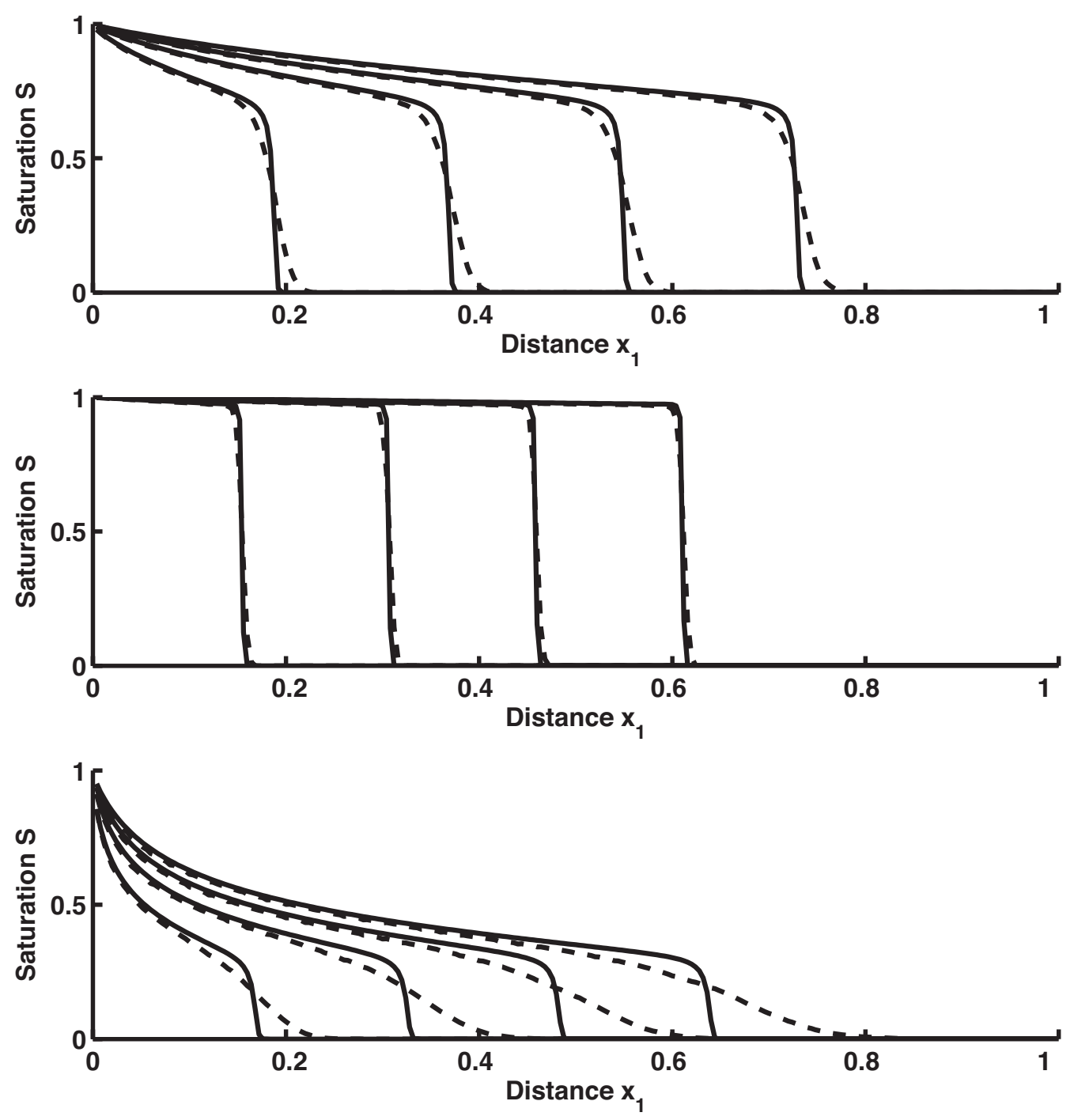

Figure 4. Average saturations for there case (top) $M=1$ and $N=-0.1$, (middle) $M=10$ and $N=-10$ and (bottom) $M=0.1$ and $N=-0.1$. Solid lines are the homogeneous numerical solutions, while the dashed lines represent the ensemble averaged heterogeneous cases 


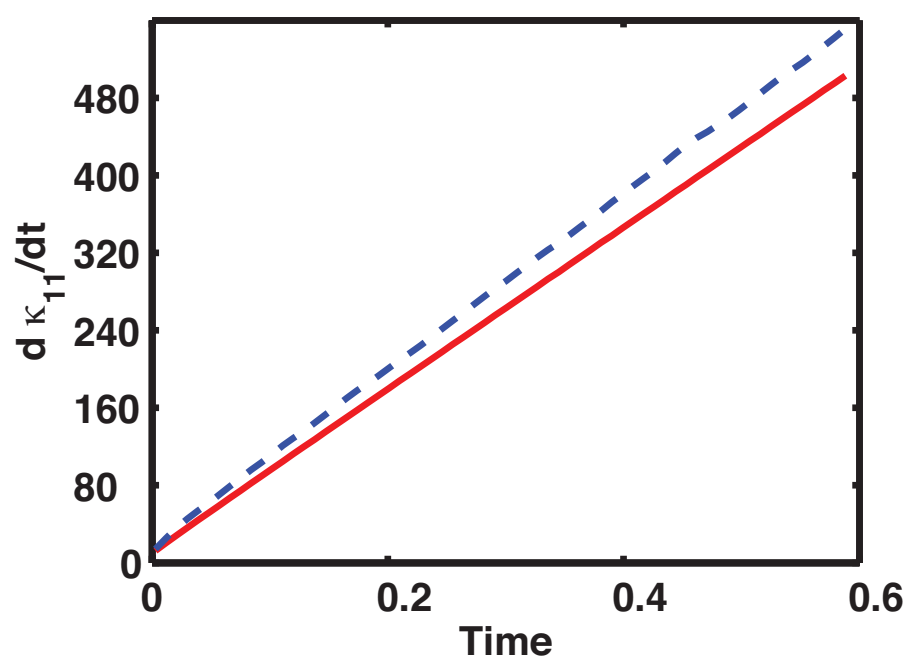

Figure 5. Illustration of the temporal Derivative of the Second Centered Moment for homogeneous (red -) and heterogeneous (blue - -) fields. The difference between these two represents the additional effect of heterogeneity, which is drawn in the following figure. For equal densities the difference between these two lines asymptotes to a constant representing the dispersion coefficient. 


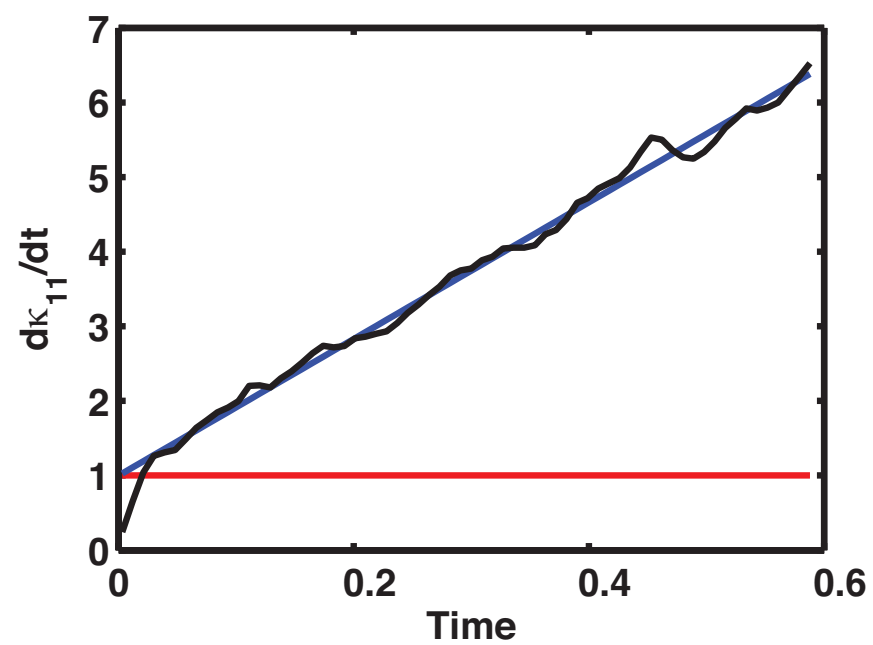

Figure 6. Heterogeneity-induced contribution to the apparent dispersion coefficient $D^{a}(t),(42)$ (nomalized so that the constant contribution to $D^{a}(t)$ is equal to one). Note the linear growth reflecting the influence of the $D^{a}$ terms, while all other terms amount to the constant dispersion coefficient case 


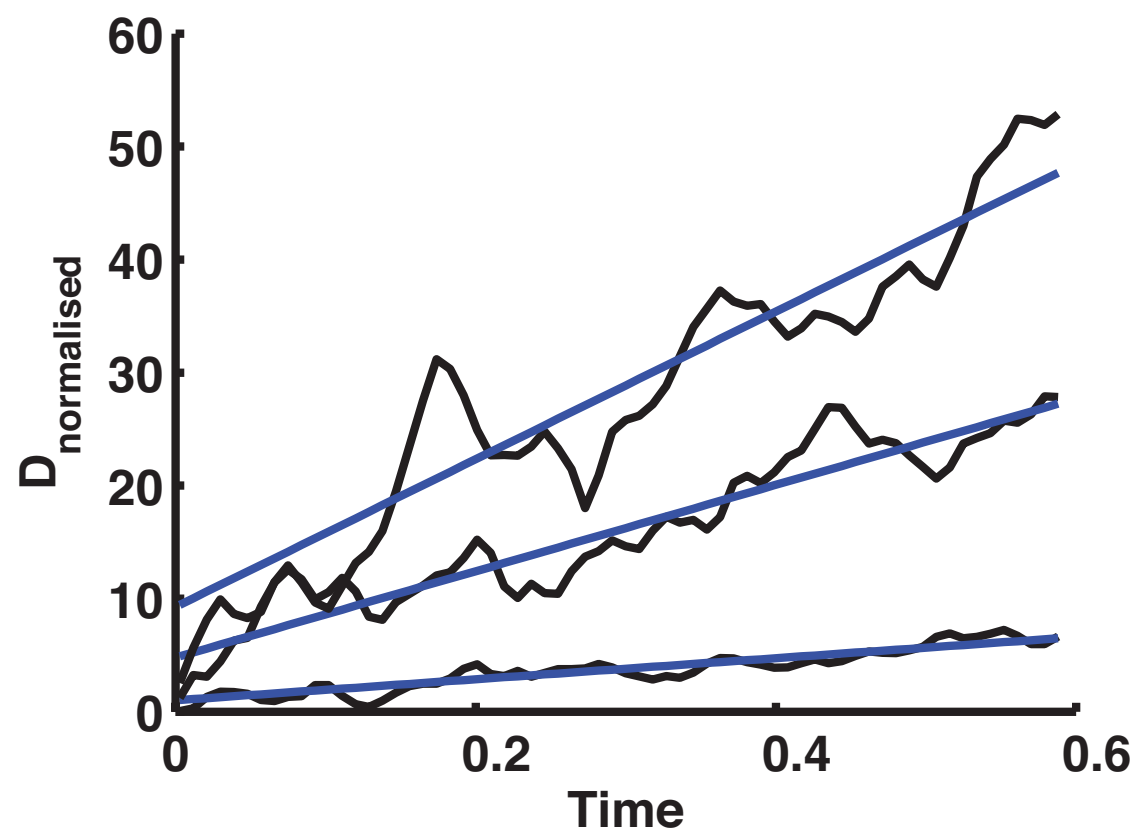

Figure 7. The normalized dispersion coefficients calculated for $M=1$ and $N=-1$ for three different variances of the permeability field $\left(\sigma_{k k}=0.1,0.5,1\right)$. In all cases the dispersion coefficient is normalized with the constant contribution associated with the $\sigma_{k k}^{2}=0.1$ case (this constant value is 1.7488 ). 


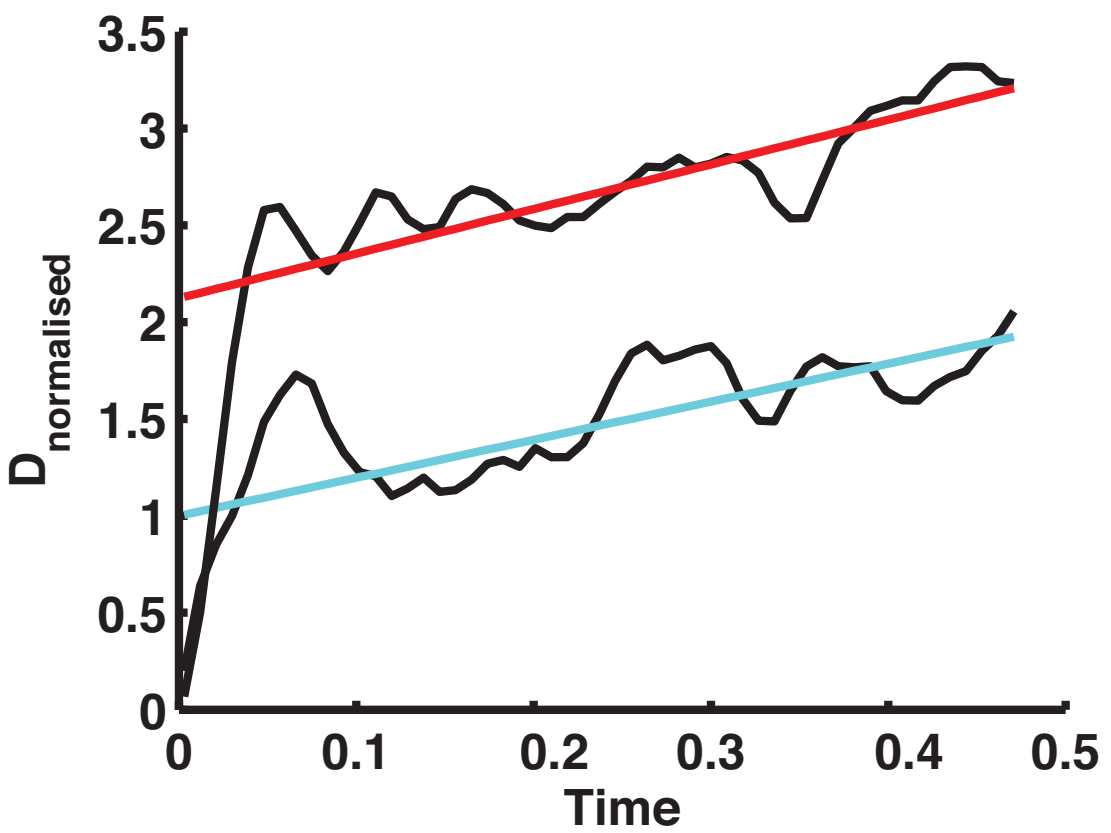

Figure 8. The dispersion coefficients calculated for two different correlation lengths of the permeability field $\left(l_{k k}=0.25,0.5\right)$. The case shown here is for $M=1$ and $N=-0.5$. The results are normalized by the constant value associated with the case $l_{k k}=0.25$, (this constant value is 7.9584$)$. 

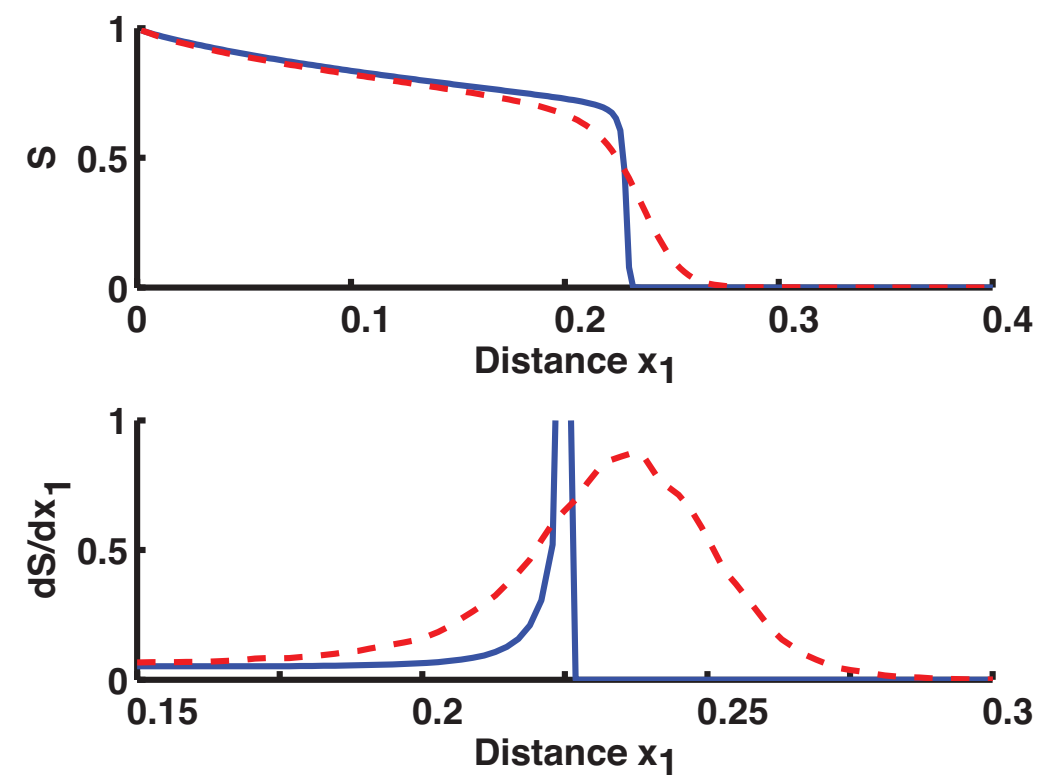

(a)

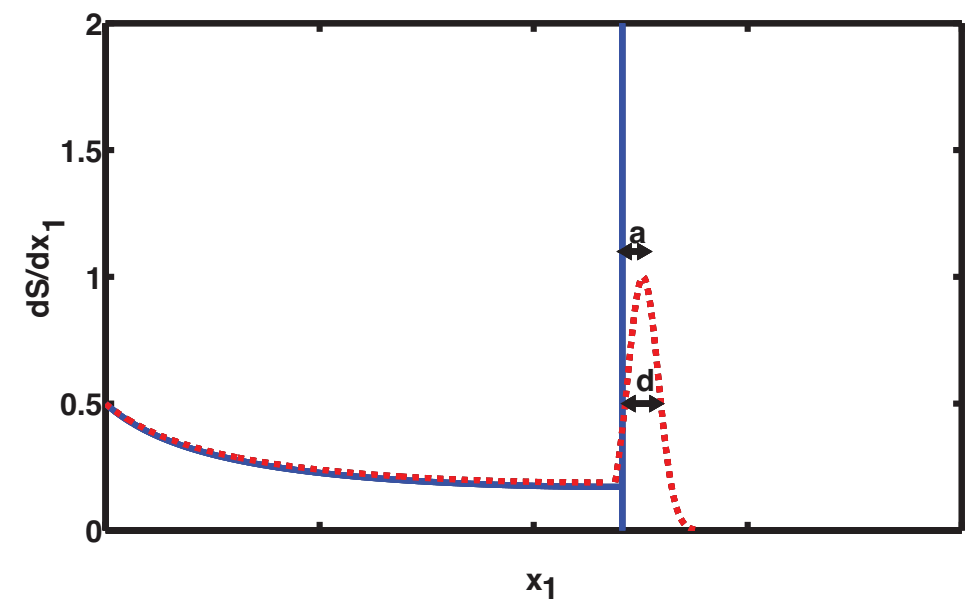

(b)

Figure 9. Derivative of Saturation. (a) Measurements from numerical simulations of saturation profiles (top) and derivatives of saturation (bottom). Here $M=1$ and $N=-1$. (b) illustrative interpretation of advective shift and dispersive spreading. In all cases he blue solid line represents the homogeneous solution and the red dashed line the heterogeneous one. 


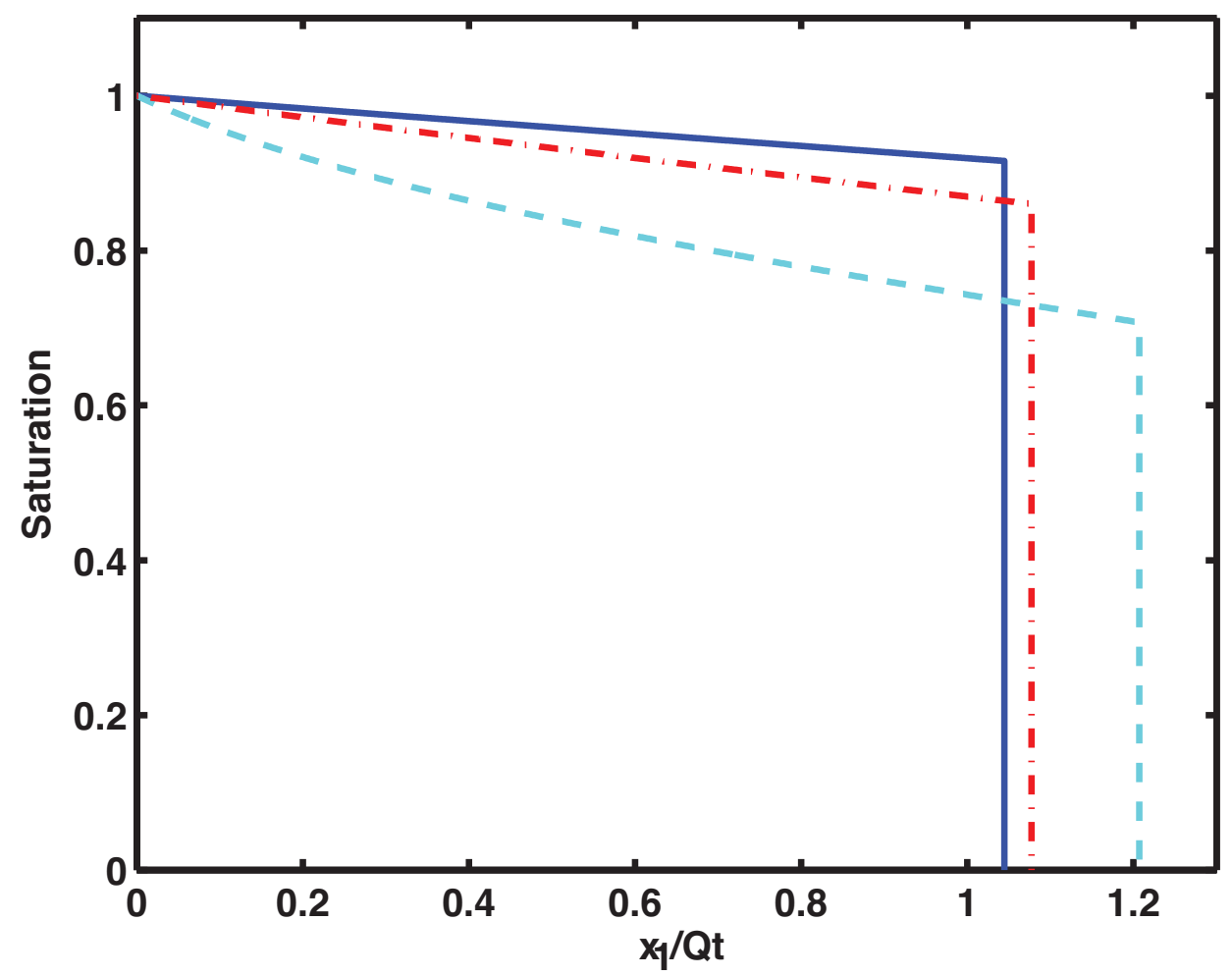

Figure 10. Homogeneous Saturation Profiles for $M=1$ and $N=0$ (light blue -), $N=-2.5($ red $-\cdot)$ and $N=-5$ (blue -). 


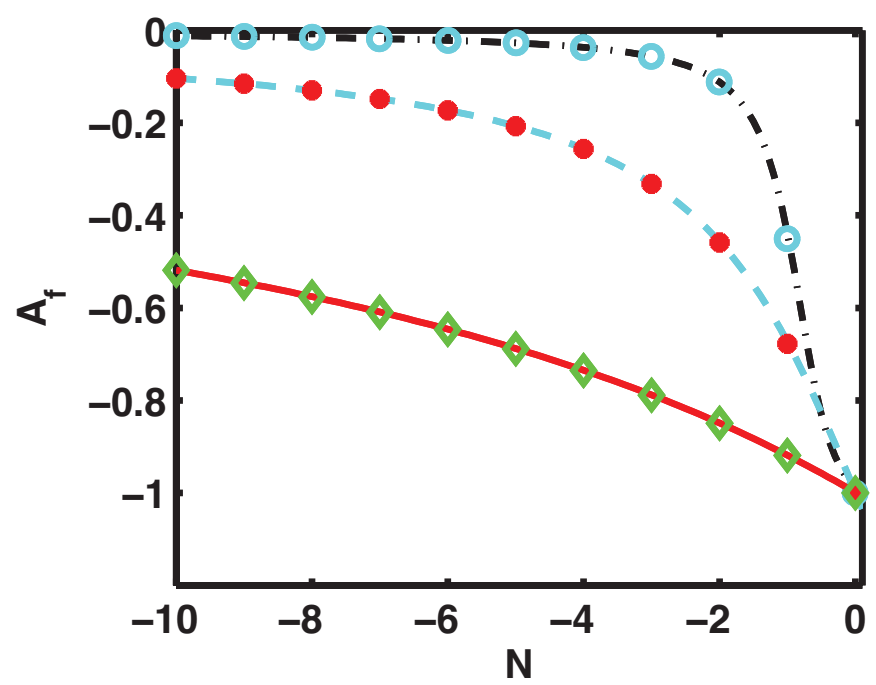

(a)

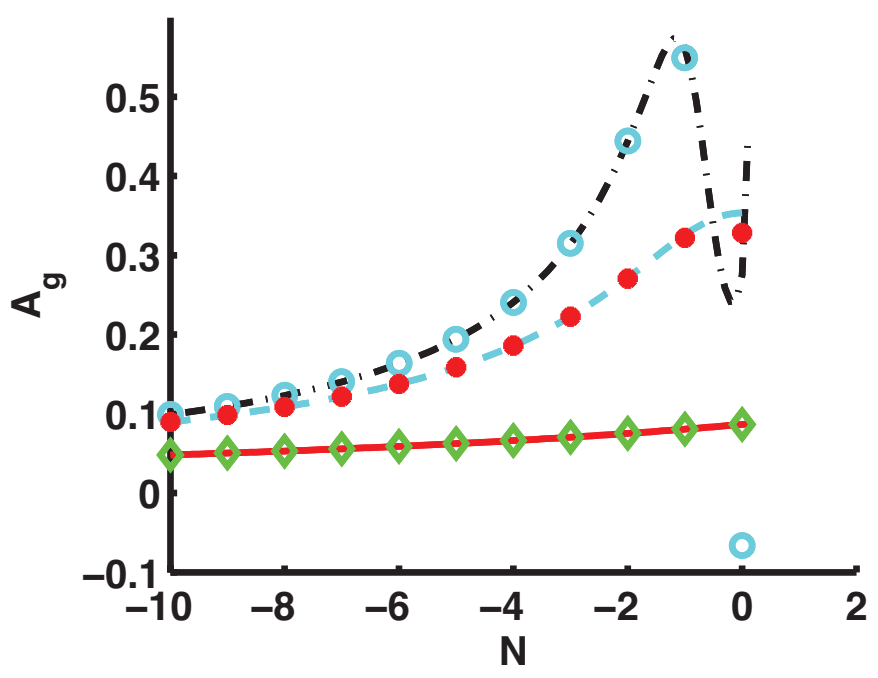

(b)

Figure 11. A comparison of the the approximate estimate of the integrals (a) $A_{f}$ and (b) $A_{g}$ based on (50) for three different values of viscosity ratio $M=0.1$ (light blue $o$ and black -.), 1 (red $\cdot$ and light blue -- ) and 10 (green $\diamond$ and red - ). The discrete points represent the values calculated with the approximation, while the continuous lines the numerically calculated value. . 